\title{
Vortex merger near a topographic slope in a homogeneous rotating fluid
}

\author{
Xavier Carton, Mathieu Morvan \\ Laboratoire d'Oceanographie Physique et Spatiale, Institut Universitaire Europeen de \\ la Mer, Universite de Bretagne Occidentale; rue Dumont d'Urville, 29280 Plouzane, \\ France; \\ emails:xcarton@univ-brest.fr, mathieu.morvan@univ-brest.fr

\section{Jean N. Reinaud} \\ Department of Mathematics and Statistics, University of Saint Andrews, North \\ Haugh, St Andrews KY16 9SS, Fife, Scotland, UK; \\ email: jnr1@st-andrews.ac.uk

\section{Mikhail A. Sokolovskiy} \\ Institute of Water Problems of the the Russian Academy of Sciences, 3 Gubkina \\ Street, 119333, Moscow, Russia; \\ email: sokolovskiy@iwp.ru

\section{Pierre L'Hegaret} \\ Meteorlogy and Physical Oceanography Department, Rosenstiel School of Marine \\ Sciences, University of Miami, 4600 Rickenbacker Causeway, Miami, FL 33149, USA; \\ email: plhegaret@rsmas.miami.edu

\section{Clément Vic} \\ National Oceanographic Center, University of Southampton, European Way, \\ Southampton, SO14 3ZH, UK; \\ email: C.Vic@soton.ac.uk
}

\begin{abstract}
The effect of a bottom slope on the merger of two identical Rankine vortices is investigated in a two dimensional, quasi-geostrophic, incompressible fluid.

When two cyclones initially lie parallel to the slope, and more than two vortex diameters away from the slope, the critical merger distance is unchanged. When the cyclones are closer to the slope, they can merge at larger distances, but they lose more mass
\end{abstract}


into filaments, thus weakening the efficiency of merger. Several effects account for this: the topographic Rossby wave advects the cyclones, reduces their mutual distance and deforms them. This alongshelf wave breaks into filaments and into secondary vortices which shear out the initial cyclones. The global motion of fluid towards the shallow domain and the erosion of the two cyclones are confirmed by the evolution of particles seeded both in the cyclones and near the topographic slope. The addition of tracer to the flow indicates that diffusion is ballistic at early times.

For two anticyclones, merger is also facilitated because one vortex is ejected offshore towards the other, via coupling with a topographic cyclone. Again two anticyclones can merge at large distance but they are eroded in the process.

Finally, for taller topographies, the critical merger distance is again increased and the topographic influence can scatter or completely erode one of the two initial cyclones. Conclusions are drawn on possible improvements of the model configuration for an application to the ocean.

\section{Keywords}

Two-dimensional incompressible flow, vortex merger, critical merger distance, bottom slope, topographic wave and vortices, diffusion.

\section{MSC 2010 numbers}

86A05, 76B47, 76E30, 76B65 


\section{Introduction}

In the oceans, vortices are long-lived features carrying water masses over long distances. In the course of their evolution, oceanic vortices can merge. Merger has been observed recently for surface vortices, in several ocean basins : in the Indian Ocean, south of the Arabian Peninsula (L'Hegaret et al., 2015), in the Atlantic Ocean, in the Bay of Biscay, and in the Pacific Ocean, near the eastern coast of Australia (see figure 1).

In the Western Arabian Sea, a large anticyclone, the Great Whirl, has been observed to merge with the nearby Socotra Eddy, along the Somali coast (Vic et al., 2014). Vortex merger in the ocean also occurs at depth: numerical simulations and in-situ observations have shown the merger of deep eddies of Mediterranean Water (meddies), near the Iberian continental shelf, in the Northeastern Atlantic ocean (Schultz-Tokos et al., 1994; Carton et al., 2010; Aguiar et al., 2013; L'Hegaret et al., 2014).

Therefore vortex merger near a coast is a common process in the ocean.

Vortex merger, in isolation from other effects, has been studied for more than three decades. It has mostly been investigated in two-dimensional incompressible fluids and in quasi-geostrophic models (Overman and Zabusky 1982; Dritschel 1985, 1986; Griffiths and Hopfinger 1987; Melander et al. 1987, 1988; Pavia and Cushman-Roisin 1990; Carnevale et al. 1991; Carton 1992; Bertrand and Carton 1993; Valcke and Verron 1993; Verron and Valcke 1994, Yasuda 1995; Yasuda and Flierl 1995; Valcke and Verron 1996, 1997; Yasuda and Flierl 1997; von Hardenberg et al. 2000; Sokolovskiy and Verron, 2000; Dritschel 2002; Reinaud and Dritschel 2002; Meunier et al. 2002; Reinaud and Dritschel 2005; Bambrey et al. 2007; Ozugurlu et al. 2008; Sokolovskiy and Carton, 2010; Sokolovskiy and Verron, 2014). These studies were performed in an unbounded fluid domain, over flat bottom.

In the two-dimensional case, two identical vortices with uniform vorticity (Rankine vortices) merge only if their initial distance is smaller than 3.3 radii. Vortex merger in stratified rotating fluids occurs on distances which may depend on stratification.

Much work has also been devoted to the dynamics of a single vortex or of multiple vortices near a topographic step or slope. In particular, point vortex dynamics was used (Meleshko, 1994; Roenby and Aref, 2010). 


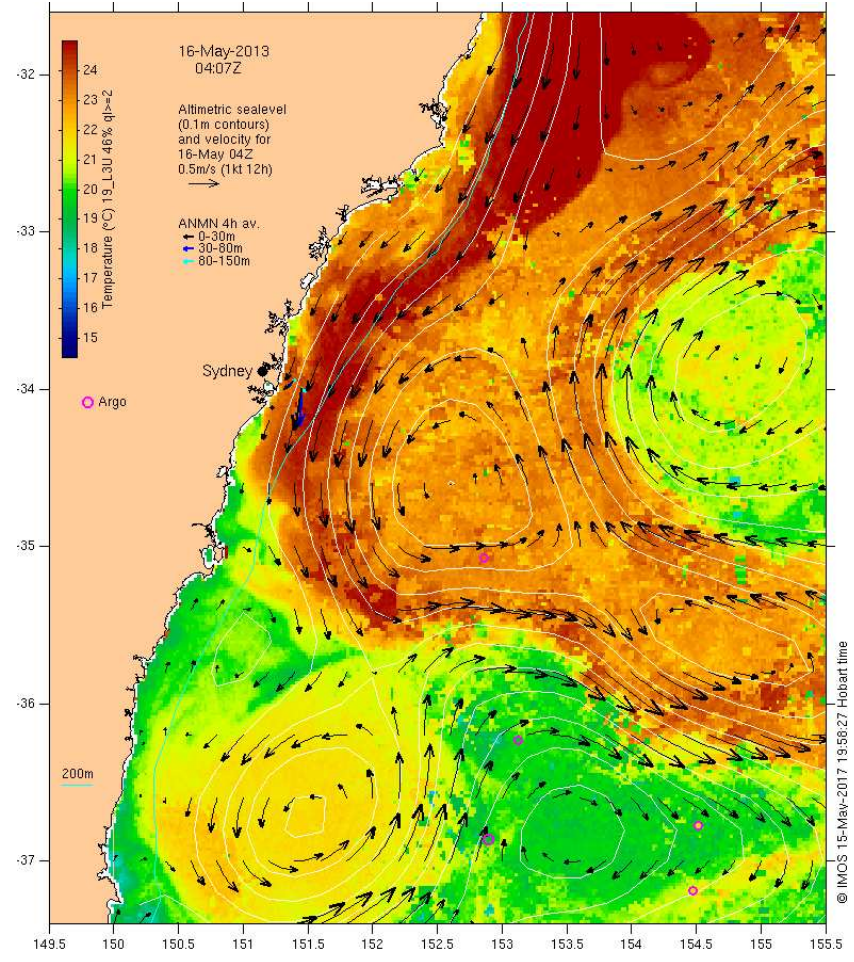

Figure 1: Sea surface temperature (color), Sea surface height (white lines) and velocity vectors showing the interaction of two warm anticyclones near the eastern coast of Australia; from IMOS Ocean Current News 
McDonald (1998) investigated analytically the motion of a point vortex near an escarpment in an equivalent barotropic, quasi-geostrophic model. He quantified the flow across the isobaths and showed that it initially leads to a repulsion of anticyclones from, and an attraction of cyclones towards the shelf. Then all vortices, whatever their polarity, drift along the shelfbreak and lose energy to the topographic waves. Vortices often stabilize at about 1.2 radii of deformation from the shelfbreak, the cyclones lying on the shelf and the anticyclones in the deep ocean. These theoretical results were confirmed by contour dynamics model simulations.

Dunn et al. (2001) extended the previous work to weak and medium intensity vortices. They showed that vortices moving against topographic waves radiate energy and move towards the shelf while vortices moving with the topographic waves can reach a steady state. Medium intensity vortices often form dipoles by extracting shelf fluid.

Hinds et al. (2007) showed that an opposite-signed vortex pair is deflected at a given angle (calculated analytically) by a shallow step, while a deep step scatters the vortex pair irreversibly.

Sutyrin and Grimshaw (2010) showed that, on a beta-plane, the interaction of a vortex with a topographic step generates secondary cyclones which can propel the vortex in a direction opposite to that given by the wall image effect. They determined that the outcome of this interaction was more sensitive to the vortex intensity and size than to other parameters.

Zhang et al. (2011) investigated the topographic waves generated by a barotropic point vortex at a shelfbreak. They studied more particularly the wave-vortex interaction, depending on the vortex polarity, and the transport of shelf water related to this interaction.

Cenedese et al (2013) studied the various interactions of a vortex in the deep ocean with a shelfbreak current depending on the ratio of vortex to current speed. They quantified the offshore transport of shelf water under the form of a filament wrapping around the vortex.

Recently, it has been shown that the interaction of a mesoscale eddy with a continental slope or a seamount, can generate smaller eddies (called submesoscale eddies; Oey and Zhang, 2004; Dewar et al., 2011; Molemaker et al., 2015; Vic et al., 2015).

But these studies did not address the effect of a topographic step, or slope, on the merger of two like-signed vortices, as it occurs in the ocean. 
The aim of this paper is to evaluate the possible merger of two identical vortices near a topographic slope, in a quasi-two-dimensional, incompressible fluid. The flow dynamics is based on the predominance of the planetary rotation over the vortex rotation (near geostrophic equilibrium). Furthermore, the vortex rotation will also dominate the topographic vorticity.

Both the critical merger distance and the processes underlying the vortex evolutions are studied. Several configurations are considered: first, two cyclones are initialized parallel to a slope. Then the influence of the vortex pair orientation, polarity, or of the topographic height are successively investigated.

Section 2 presents the model and configurations. Section 3 introduces the vortex interaction with a qualitative analysis. Section 4 focuses on vortex merger near a low topography. Section 5 is devoted to the influence of the topographic height. Finally conclusions are drawn in section 6 .

\section{The model}

\subsection{Model equations}

In the most general case, the barotropic shallow-water, potential vorticity equation governs the evolution of incompressible, homogeneous fluid flows, with flat upper surface, and prescribed bottom topography (whatever its height), and with uniform external rotation:

$$
\partial_{t} q+\vec{u} \cdot \vec{\nabla} q=\nu_{4} \nabla^{4} \omega
$$

where $q=(\omega+f) / h$ is potential vorticity, $\omega=\partial_{x} v-\partial_{y} u$ is relative vorticity, $f$ is the Coriolis parameter (twice the external rotation rate) considered constant here $f=f_{0}$. $h=H-h_{b}(x, y) \geq 0$ is the local fluid thickness. The maximal fluid depth is $H$ and $h_{b} \geq 0$ is the height of topography.

The right-hand side of equation (1) is biharmonic dissipation of vorticity, implemented numerically to remove vorticity accumulation at small scales.

The numerical hyper viscosity is very weak (see below; $\nu_{4}=10^{-9}$ ). 
The thickness (or continuity) equation is

$$
\partial_{t} h+\vec{\nabla} \cdot(h \vec{u})=0 .
$$

Here, considering a flat upper surface, $\partial_{t} h=0$. Therefore $h u=-\partial_{y} \psi$ and $h v=\partial_{x} \psi$, where $\psi$ is the transport streamfunction. Then, $\omega=\vec{\nabla} \cdot[(\vec{\nabla} \psi) / h]$. Since $h$ and $f_{0}$ are prescribed, $q$ is also a function of $\psi$ only.

Equation (1) can be written

$$
\partial_{t} \omega+J(\psi, q)=\nu_{4} \nabla^{4} \omega
$$

where $J(f, g)=\partial_{x} f \partial_{y} g-\partial_{x} g \partial_{y} f$ is the Jacobian operator.

When the elevation of the bottom topography $h_{b}$ is small compared to the total fluid depth $H$, and when $\omega / f_{0} \ll 1$, equation (2) becomes the quasi-geostrophic potential vorticity equation

$$
\partial_{t} \omega+J(\phi, Q)=\nu_{4} \nabla^{4} \omega
$$

where $\phi=\psi / H$ is the streamfunction, $\omega=\nabla^{2} \phi$ and $Q=\omega-f_{0} h_{b} / H$ is quasigeostrophic potential vorticity.

\section{$2.2 \quad$ Numerical model}

Equation (3) for the quasi-geostrophic model is implemented numerically in a square, biperiodic domain, using a pseudo-spectral technique spatially and a mixed EulerLeapfrog scheme temporally. The time step is bounded by the Courant-Friedrich-Levy condition. The domain length and width are $L=4 \pi$ (with $x, y \in[-2 \pi, 2 \pi]$ ). Parametric studies are performed with 256 collocation points in the model, while detailed studies used 512 collocation points. Very weak biharmonic viscosity $\left(\nu_{4}=10^{-9}\right)$ is applied in the model. This viscosity does not alter the physical results of the model and only removes small scale noise. By doubling the domain size for the study of distant vortices, we check that the image effect due to periodicity does not qualitatively alter the two-vortex evolutions (fine details may change, but not the global evolution).

The initial conditions of this model are a pair of identical, circular vortices of radius 
$R=0.5$, enclosing uniform quasi-geostrophic relative vorticity $\nabla^{2} \phi=1$, and lying initially at a distance $d$ from each other, and at a distance $d_{c}$ from the topography (see figure $2 \mathrm{~b}$ ). Elsewhere, $\nabla^{2} \phi=0$.

For an application to the ocean, we consider vortices in the Arabian Sea; they have a peak velocity $V \sim 0.4 \mathrm{~m} / \mathrm{s}$ at a radius $R \sim 30 \mathrm{~km}$. Therefore, length and time are scaled between the model and the ocean via $L=610^{4} \mathrm{~m}$ and $T=410^{4} \mathrm{~s}$. An estimate of $\omega$ is $2 V / R=2.710^{-5} s^{-1}$. In the Arabian Sea, $f_{0}=5.410^{-5} s^{-1}$, so that $\omega / f_{0}=0.5$. The Rossby number is therefore $R o=0.25$.

In the model, the dimensionless value of the Coriolis parameter is $f_{0}=2$, which scales the topographic vorticity $f_{0} h_{b} / H$ in equation (3).

The bathymetry is a smooth slope, with a deep domain at the center (near $y=0$ ), and shallow domains both north and south (for positive and negative $y$ ):

$$
h_{b}(y)=h_{b 0}\left[1.0+0.5 \tanh \left(\left(y-y_{t}\right) / L_{t}\right)-0.5 \tanh \left(\left(y+y_{t}\right) / L_{t}\right)\right]
$$

We have $h_{b 0}=0.2 H$ for most of this study (section 4). Finally, in the model, we set $H=1.0$.

Numerically, the choice of topographic parameters is, for anticyclones, $y_{t}=5.0, L_{t}=0.5$ (since anticyclones move away from the slope into the deep region), and for cyclones, $y_{t}=2.5, L_{t}=0.5$ (since cyclones move in the opposite direction).

To track fluid masses, a few (discrete) particles are seeded in various parts of the domain initially (with tags indicating their origin). Their advection illustrate the vortex trajectories. They also show how the periphery of a vortex can be eroded during the two-vortex interactions.

A passive tracer, with continuous distribution, is used to evaluate the mixing of shallow and deep fluid masses. This tracer is initialized as a smooth front over the topography.

$$
T(x, y, t=0)=T_{0}\left[\tanh \left(\left(y-y_{t}\right) / L_{t}\right)-\tanh \left(\left(y+y_{t}\right) / L_{t}\right)\right]-T_{a v g}
$$

with $T_{0}=1$. The model being biperiodic (and using Fourier transforms spatially), substracts the spatial average from all calculated fields, hence the term $-T_{a v g}$ in the equation above. 


\section{Vortex interaction near a topographic slope : qual- itative analysis}

In this section, we present a qualitative analysis of the interaction of a vortex with the slope topography, based on the conservation of vorticity and on the associated motion of the vortex patches.

A single cyclone, close to a southern topographic step (or slope), extracts fluid from the shelf (shallow region), and advects it to its east; this fluid thus gains positive relative vorticity, see figure 2 a. The cyclone pushes fluid shoreward to its west, producing a negative vorticity anomaly there, see figure 2 a (left). This double vorticity anomaly (called secondary vorticity poles) advects the cyclone shoreward (southeastward).

These secondary vorticity poles result from with the development of a topographic Rossby wave, propagating eastward along a southern step/slope. This wave both advects and deforms any neighboring vortex. As a first approach to this mutual influence, a simple model featuring the interaction of two point-vortices and a topographic vorticity front, is presented in appendix 1.

For two cyclones, such vortex motion and deformation must be combined with the co-rotation, or inward spiraling motion, of the vortex pair itself.

Therefore, a cyclone pair starts co-rotating and drifting towards the step. This, in turn, triggers topographic Rossby waves along the step. These waves may advect the two vortices closer to each other and thus may favor merger. But also, the velocity shear associated with these waves can elongate the two vortices and fragment them. This splitting process can compete with the merger of the vortices, and thus weaken its efficiency 1 . We need to determine numerically which of these two effects prevails. This depends on the velocity and velocity shear induced on any primary vortex, by the other primary vortex and by the secondary vortices.

For anticyclones, the situation is different :

A single anticyclone is advected offshore (northwestward), by the secondary vorticity poles, when initially located near a southern topographic step, see figure 2 a (right).

\footnotetext{
${ }^{1}$ Efficiency is the ratio of final (after merger) to initial circulation of the vortices
} 


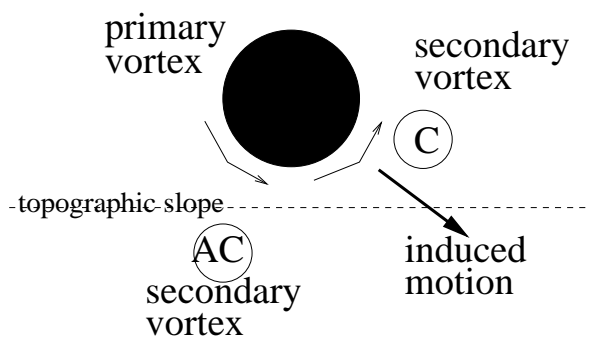

a) CYCLONE NEAR SLOPE

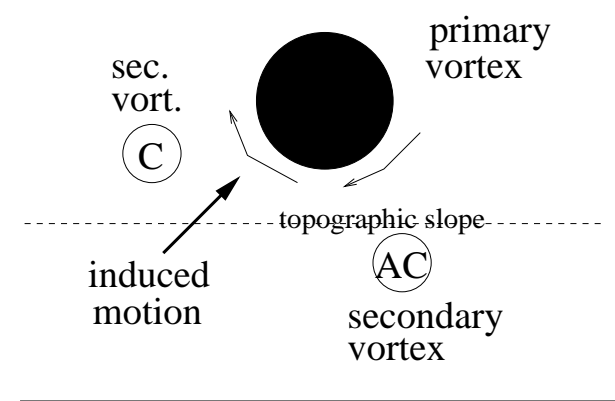

ANTICYCLONE NEAR SLOPE

ocean

surface $\quad L_{t}$ : half width of topographic slope $\mathrm{y}_{\mathrm{t}}$ : distance of the slope to the center of the domain

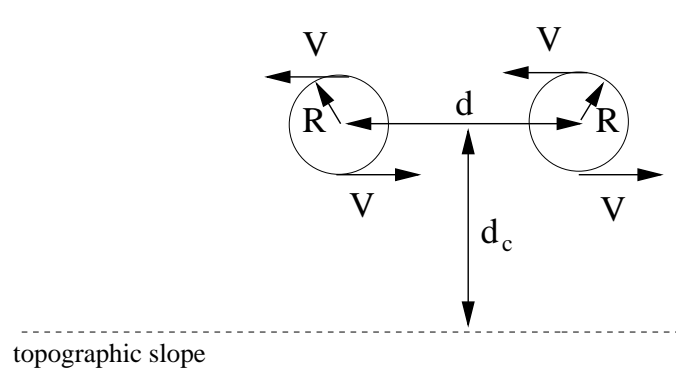

TWO CYCLONES NEAR A SLOPE (TOP VIEW)

b)

$\mathrm{R}$ : vortex radius $\mathrm{V}$ : vortex azimuthal velocity $\mathrm{d}$ : distance between vortex centers $\mathrm{d}_{\mathrm{c}}$ : distance from vortex axis to the topography

Figure 2: (a) left: interaction (viewed from the top) of a single cyclone with a topographic slope, forming secondary vorticity poles leading to the advection of the primary vortex towards the slope; right: corresponding situation for a single anticyclone in the presence of the slope: the secondary vortices advect the primary vortex away from the slope; (b) Upper plot: Meridional cross-section of the basin showing the bottom topography; lower plot: top view of the initial situation of two cyclones along the topographic slope, with the physical parameters. 


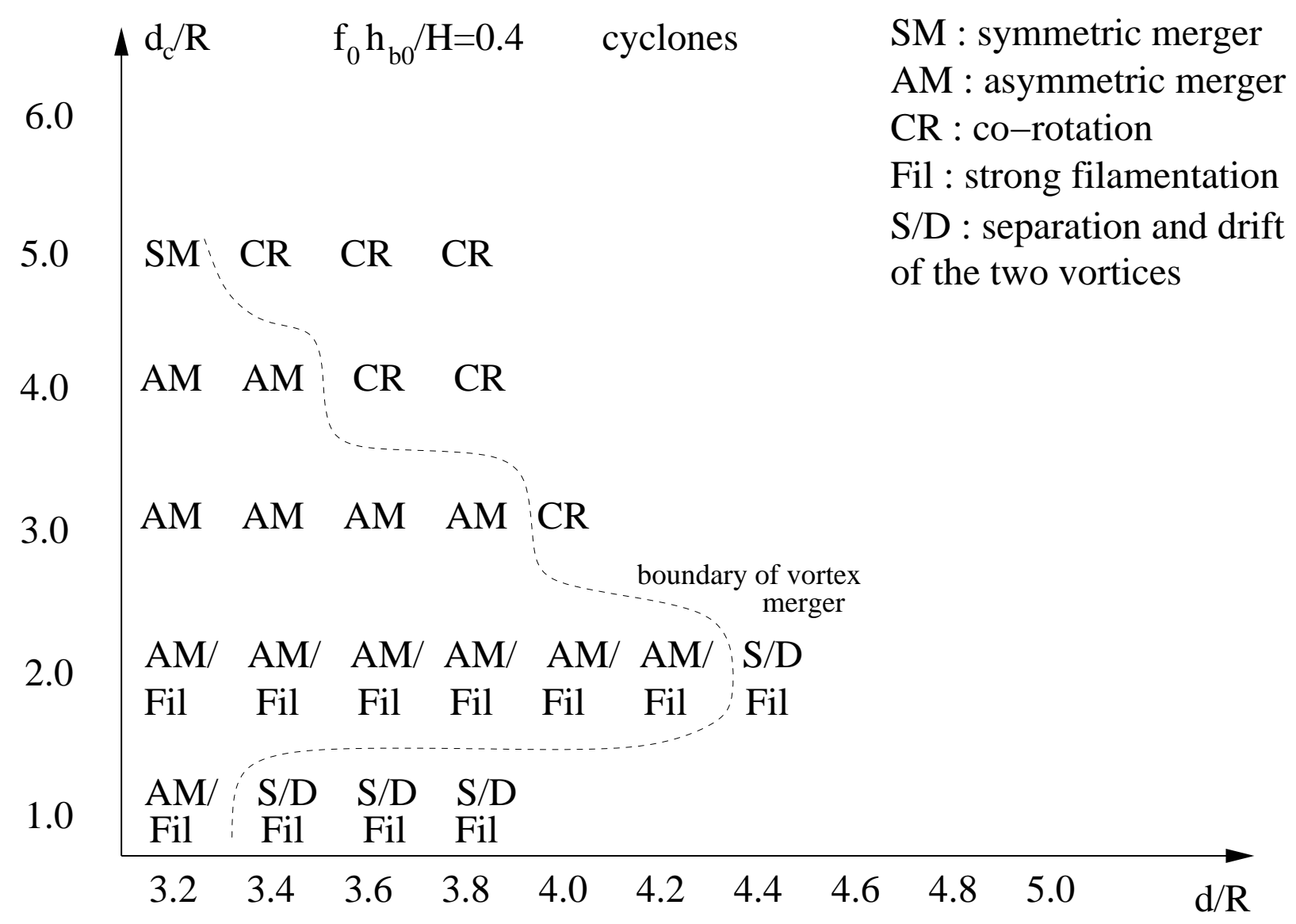

Figure 3: Nonlinear regimes of two cyclone interaction, with respect to their initial separation (scaled by their radius), $d / R$, and to their initial distance to the slope $d_{c} / R$. These cyclones are initially parallel to the topographic slope. The topographic height, in terms of vorticity, is $f_{0} h_{b 0} / H=0.4$. The various regimes are indicated in the figure. 
Two anticyclones start rotating in the clockwise direction; the easternmost anticyclone becomes the closest to the step and hence feels the influence of the cyclonic topographic vorticity. This topographic vorticity pole can couple with this anticyclone as a dipole. This dipole can drift offshore, towards the other anticyclone. Then, the two anticyclones can merge.

Thus, qualitatively, the merging process may be favored by a topographic step, for anticyclones.

Again, this has to be studied numerically to obtain quantitative results.

\section{Numerical simulations of the evolution of a vortex pair}

In this section, we consider the motion of two vortices near a topographic slope with $f_{0} h_{b 0} / H=0.4$, compared to the unit vortex vorticity $\omega=\nabla^{2} \phi=1$.

\subsection{Regime diagram for a cyclone pair initially parallel to the slope}

Figure 3 shows the various nonlinear regimes for the interaction of two cyclones near a southern step, obtained by analysing the outcome of our numerical simulations.

When the two cyclones are distant from the step $\left(d_{c} / R=5\right)$, the topographic effect is negligible and the critical merger distance lies between $d / R=3.2$ and $d / R=3.4$, as it is well known for two Rankine vortices, in the absence of other effects.

As the vortices are initially located closer to the step, the merging process becomes more asymmetric and less efficient; the final vortex does not gather all the mass of the initial two vortices. Indeed, the easternmost cyclone rotates in the anticlockwise direction (away from the step) and is thus less affected by topographic Rossby waves. The westernmost cyclone is advected towards the step, into the shallower region, and is elongated. A large part of it is absorbed (via merger) in the easternmost cyclone. The remainder is cast away as filaments and progressively scattered and dissipated. The elongation of the westernmost cyclone explains why it can interact more easily with its partner, and thus why the critical merger distance increases, as $d_{c} / R$ decreases. The 
vortex filamentation over topography explains why the merging efficiency decreases, compared with merger over flat bottom.

For small $d_{c} / R$, the vortex deformation is so intense and rapid that the merging process most often cannot take place. The outcome in such a situation is often separation, drift and shearing out of the vortices in the shallower region. Therefore, the critical merger distance decreases then to about $d / R=3.2$.

Finally, it must be mentioned that vorticity remains mostly confined near the step or in the shallow region. Therefore, the fluid exchange is mostly confined there.

To sum up, two cyclones near a step undergo merger more easily as they are closer to the step, but this process becomes less efficient. But two cyclones very close to the step are separated and do not merge.

\subsection{Analysis of two nonlinear regimes}

\subsubsection{Evolution of vorticity and of deformation}

We study in more detail two nonlinear regimes associated with the presence of bottom topography. The simulations are run with 512 nodes in each direction.

Firstly, we present the case $d_{c} / R=2, d / R=3.2$ for which asymmetric merger occurs, followed by the deformation of the vortices.

Figure 4 shows the time evolution of relative vorticity.

The two cyclones lie initially at the bottom of the topographic slope (figure 4a). Their co-rotation rapidly brings the (initially) westernmost one over the slope (figure 4b). This cyclone is strongly elongated and then sheds filaments (figure 4c). Therefore, only a part of it is absorbed into the (initially) easternmost cyclone (figure 4 $\mathrm{d}$ ). The total circulation of the merged vortex is about $75 \%$ of the sum of the two initial circulations, while the negative (topographic) circulation amounts to about $35 \%$ of this sum.

At a much later time, even this latter cyclone is strongly deformed by the shear induced by the topographic vortices (figure 44). It splits and the final circulation is then only $35 \%$ of the total initial circulation. 


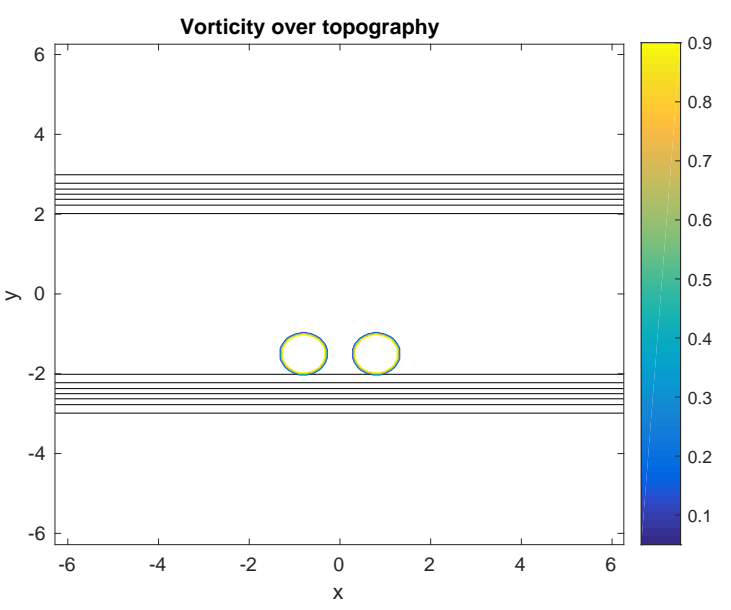

a)

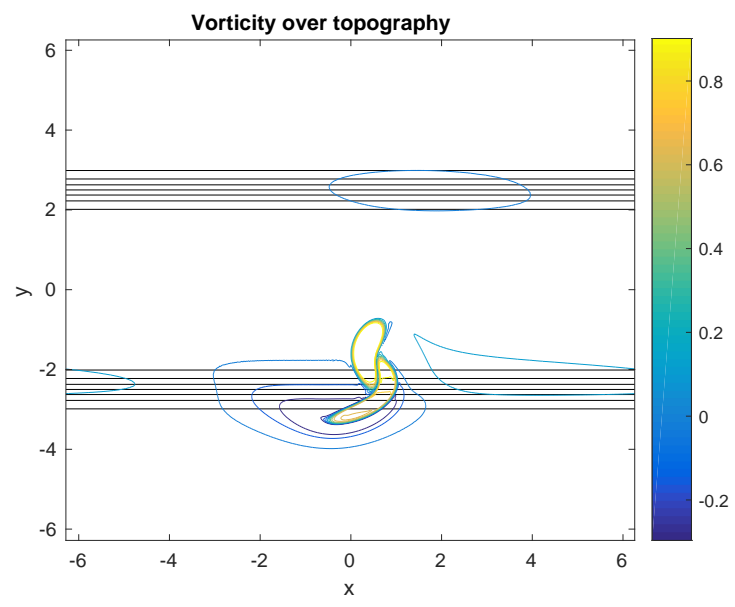

c)

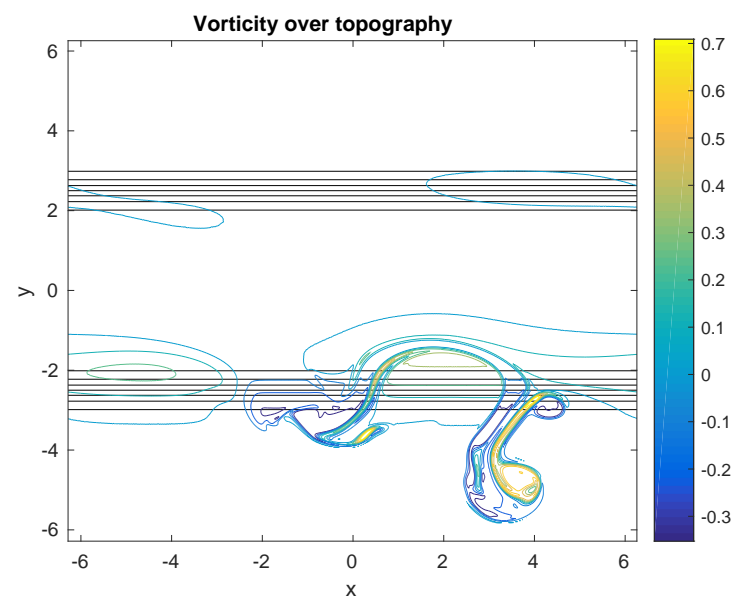

e) b)

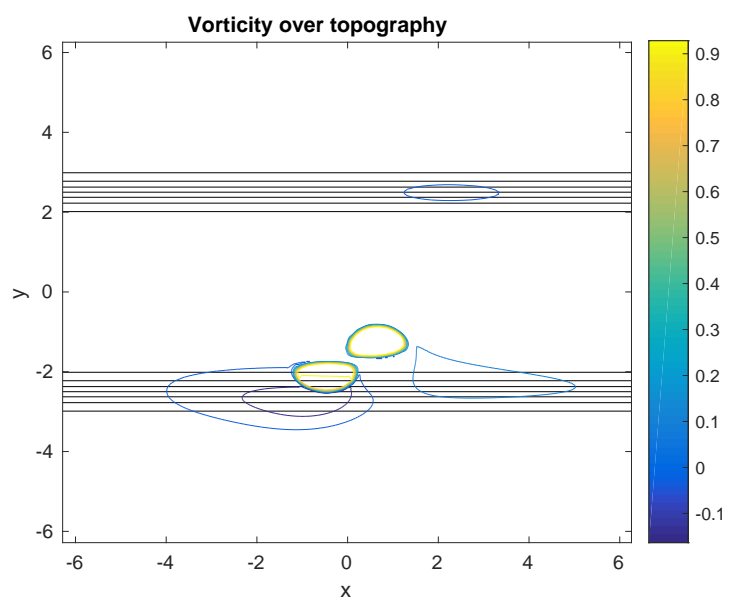

d)

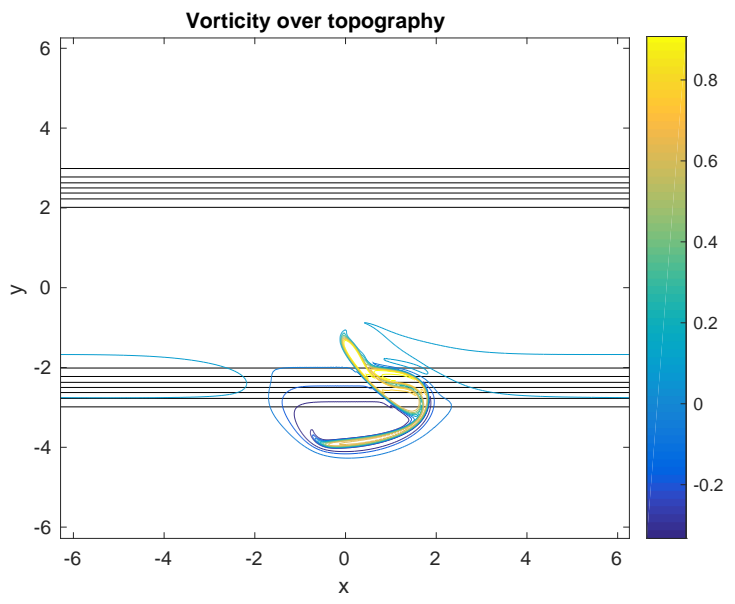

Figure 4: Time evolution of vorticity maps for two cyclone interaction with normalized initial distance to the shelf $d_{c} / R=2$, and initial distance between vortices $d / R=3.2$. Times shown are $t=0,6,12,18,60$ (subplots a, b, c, d, e). We remind that the vortex turn-over time $\tau$ is $4 \pi$. The horizontal black lines are isobaths (indicating the bottom slope), and the color scale characterizes the4intensity of the relative vorticity. 


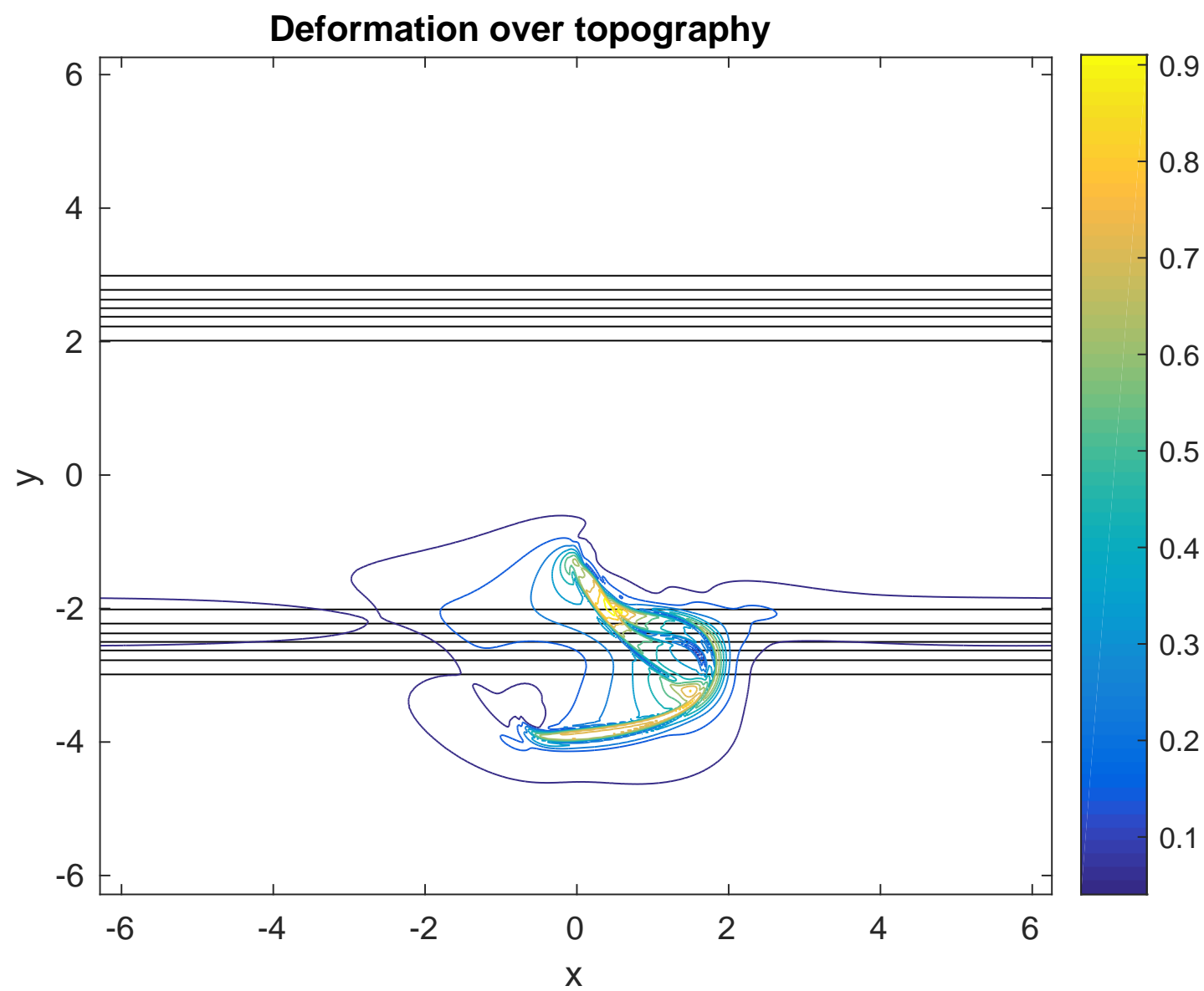

Figure 5: Deformation map for two cyclone interaction with $d_{c} / R=2, d / R=3.2$. Time shown is $t=18$ and $\tau=4 \pi$ is the vortex turn-over time. The horizontal black lines are isobaths (indicating the bottom slope), and the color scale characterizes the intensity of the rate of deformation. 
a)

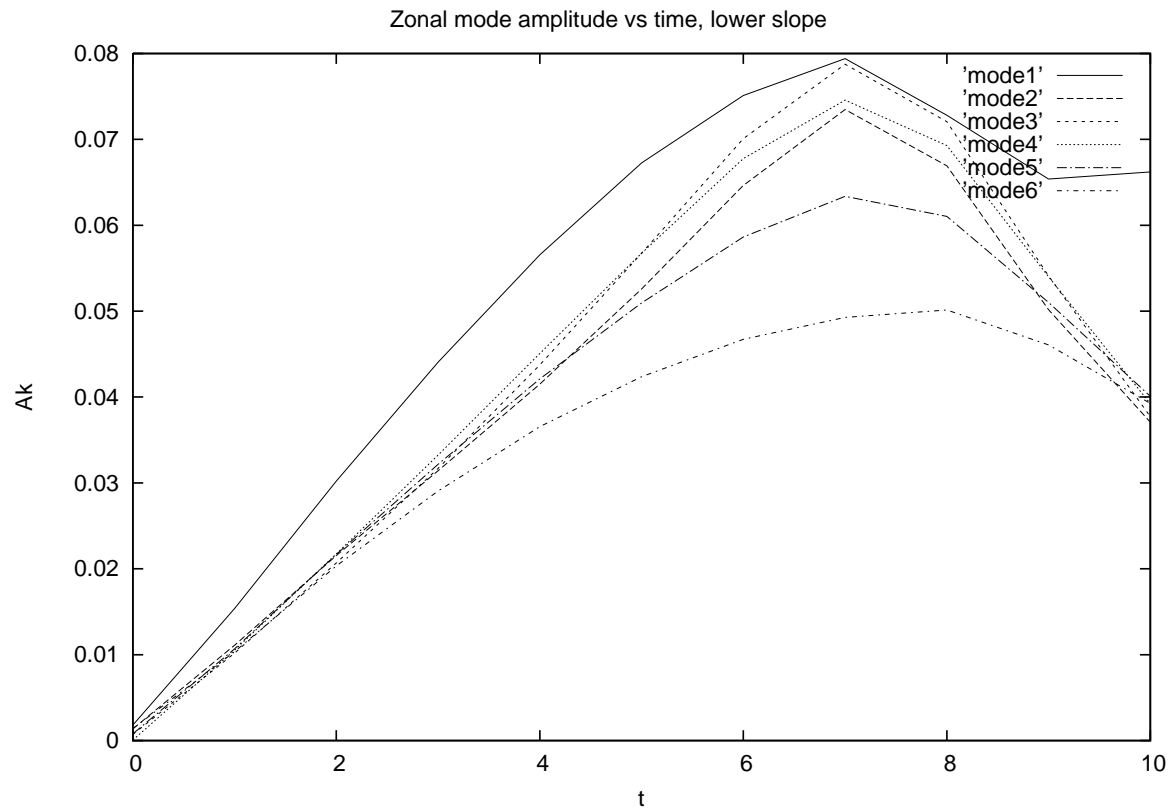

b)

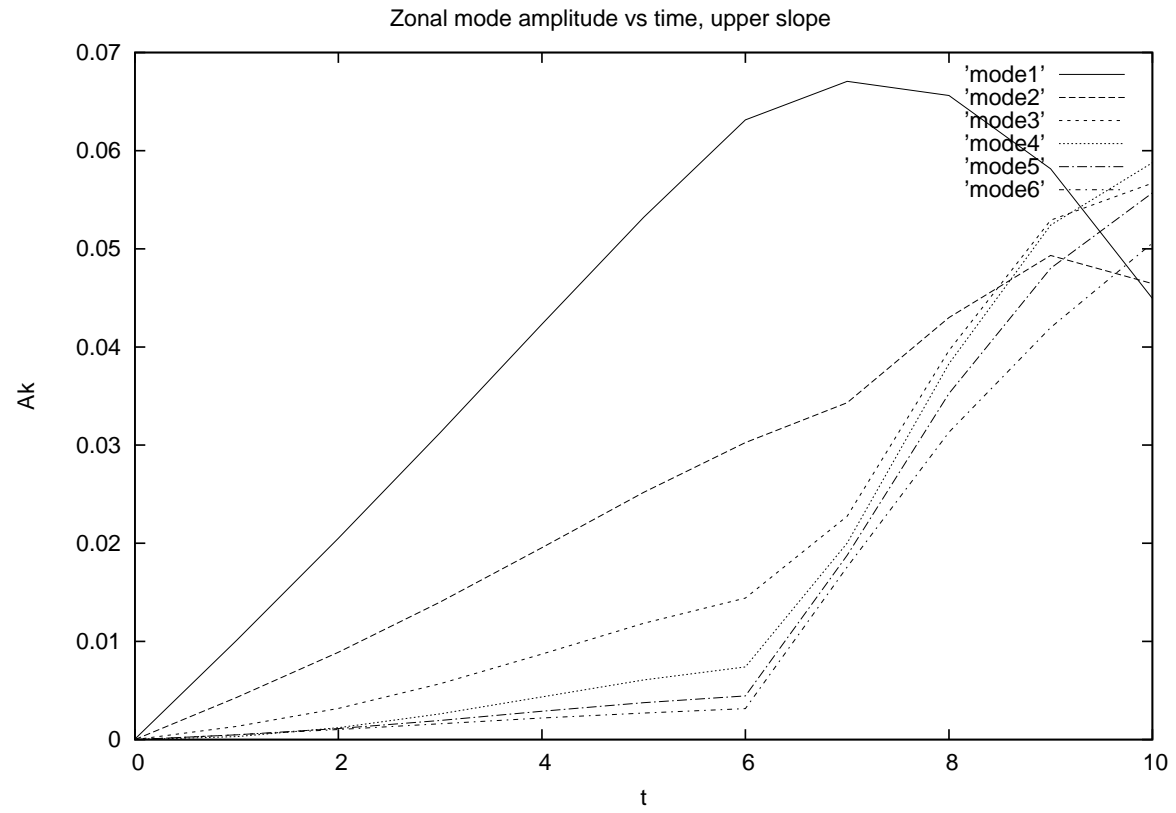

Figure 6: Amplitude of topographic Rossby wave, (a) at the lower slope, (b) at the upper slope, during the two cyclone interaction with $d_{c} / R=2, d / R=3.2$. Zonal modes $k=1,2,3,4,5,6$ are shown. 
Figure 5 shows the rate of deformation $\sigma$ of the two cyclones over the topographic slope. This rate is defined by

$$
\sigma^{2}=\sigma_{1}^{2}+\sigma_{2}^{2}, \quad \sigma_{1}=\partial_{x} u-\partial_{y} v, \quad \sigma_{2}=\partial_{x} v+\partial_{y} u
$$

Clearly, deformation is strong at the edges and tip of the vortices, where filaments are produced.

The generation of topographic Rossby waves is analyzed at the early stage of this simulation, via a Fourier transform of the vorticity field, over the upper and lower parts of the slope (i.e. for $y_{t}+L_{t}<y<y_{t}$ and for $y_{t}<y<y_{t}+L_{t}$, figure 6). Over the lower slope, waves with all wavenumbers from $k=1$ to $k=6$ grow from the start. This corresponds to the contribution of the wave and of the vortex pair, which produces filaments and small-scale features. On the upper half, only the wave is present at early times. Then long waves dominate. Indeed, the Fourier transform of the vorticity interface deviation for two point vortices and a topographic front, has an amplitude proportional to $\exp \left(-k d_{c}\right)$ (see appendix 1). Therefore, shorter waves have smaller amplitude.

The effect of these waves on the two vortices are as follows:

- the fluid climbing on the slope acquires negative relative vorticity and conversely for the fluid descending from the shallow region ;

- they accelerate the westernmost cyclone towards the shallow region and elongate it; they slow down the rotation (and offshore motion) of the easternmost cyclone;

- this influence induces a decrease in the distance between the two initial cyclones, favoring their merger.

Again, this explains why two cyclones can merge at larger distance than in the absence of topography, but one cyclone being strained, merger becomes asymmetric and less efficient.

Secondly, we present the case $d_{c} / R=1, d / R=3.8$ for which the vortices do not merge, but only filament.

The two cyclones lie initially at the bottom of the topographic slope. The time series 


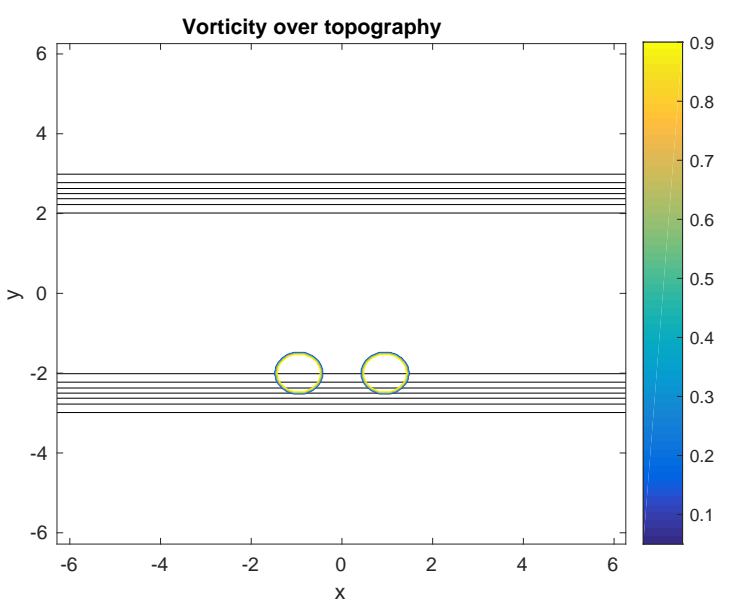

a)

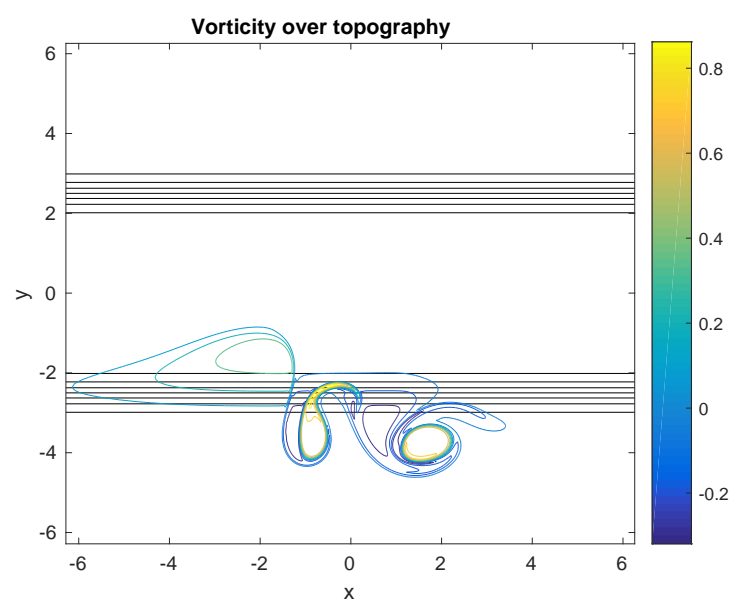

c)

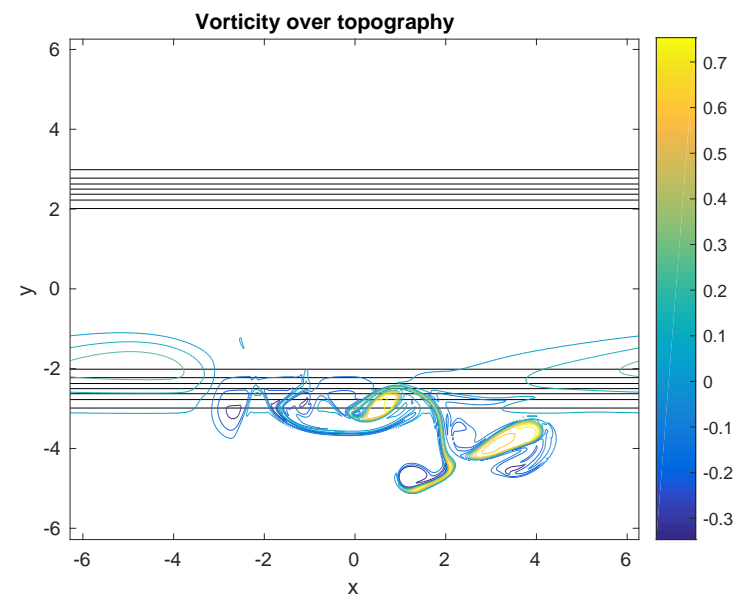

e) b)
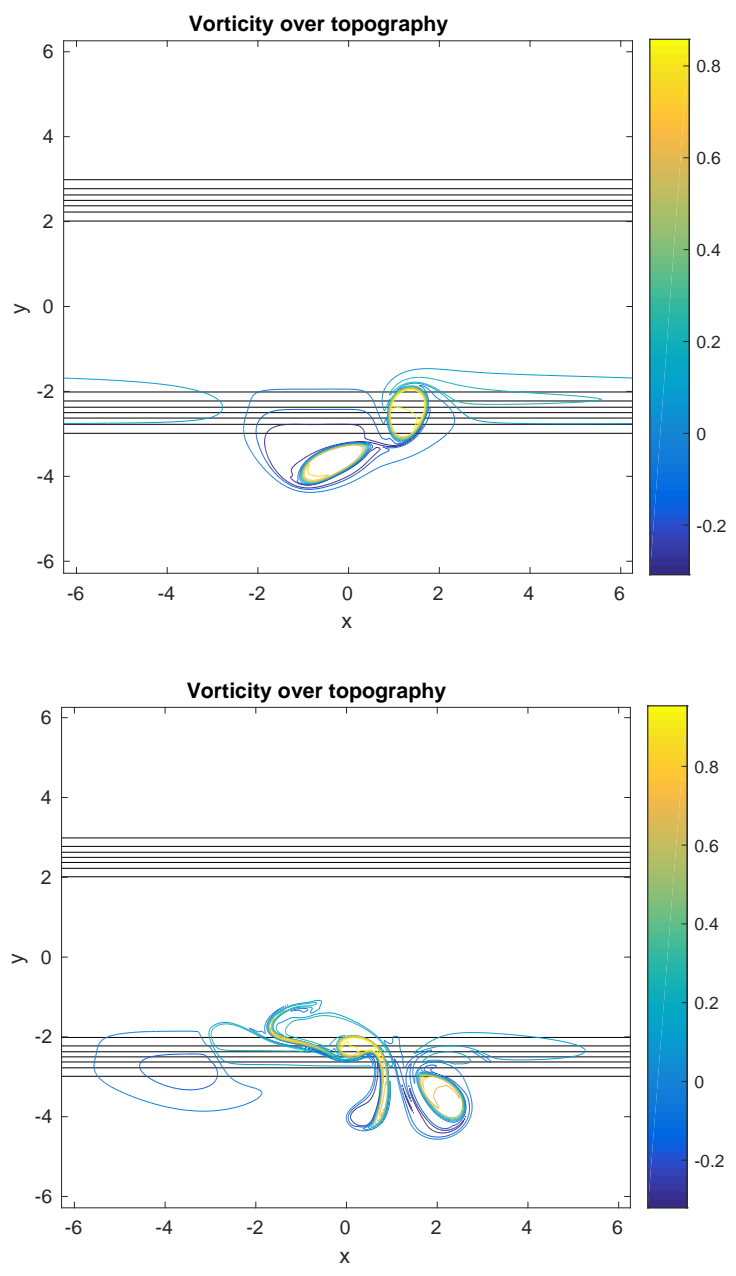

d)

Figure 7: Time evolution of vorticity maps for two cyclone interaction with $d_{c} / R=1$, $d / R=3.8$. Times shown are $t=0,15,30,45,60$ (subplots a,b,c,d,e). We remind that the vortex turn-over time $\tau$ is $4 \pi$. The horizontal black lines are isobaths (indicating the bottom slope), and the color scale characterizes the intensity of the relative vorticity. 


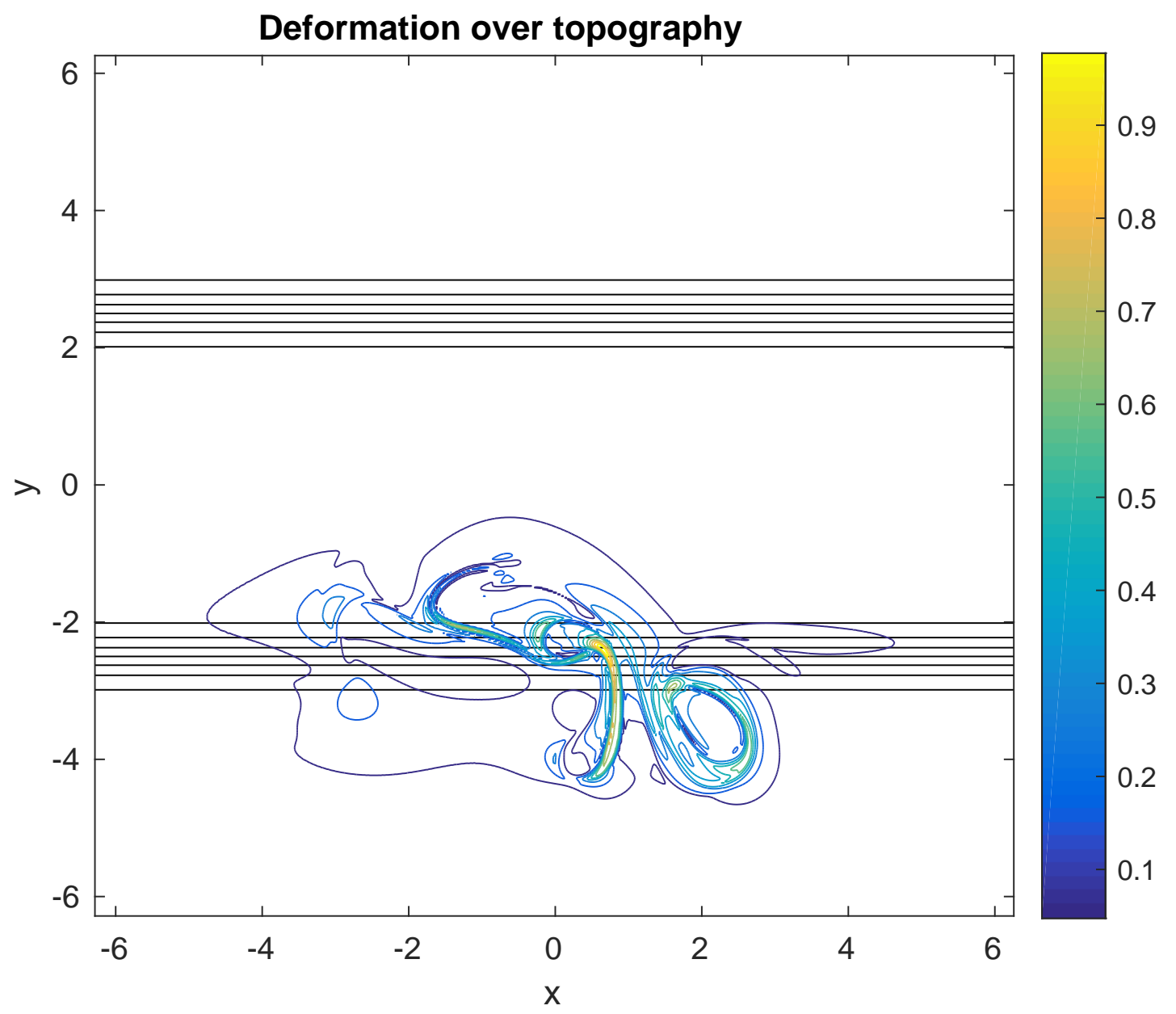

Figure 8: Deformation map for two cyclone interaction with $d_{c} / R=1, d / R=3.8$. Time shown is $t=45$ and $\tau=4 \pi$ is the vortex turn-over time. The horizontal black lines are isobaths (indicating the bottom slope), and the color scale characterizes the intensity of the rate of deformation. 
of vorticity maps (figure 7) indicates that the westernmost cyclone climbs onto the shelf and filaments at its northern tip, while the easternmost cyclone climbs onto the slope, essentially advected by the topographic wave (which circles around the vortex). Then, the two cyclones lie on the shelf where they interact but do not merge, and finally the westernmost cyclone totally filaments. This strong deformation concentrated on the western cyclone clearly appears in figure 8 ,

Over the upper slope, the topographic wave analysis (figure not shown) indicates that all wavelengths grow initially, but the long waves dominate at longer time. Over the lower part of the slope, the wave amplitudes decrease because the vorticity field initially encompasses the two cyclones which finally drift southward and lie over the shelf.

\subsubsection{Particle trajectories}

In the case $d_{c} / R=2, d / R=3.2$, a few discrete passive particles are seeded in the domain (see figure 9). The evolution of the two cyclones in this case, is partial and asymmetric merger, followed by drift and shearing and straining.

The aim of particle tracking here, is not to perform statistical studies of dispersion, but to follow the vortices, and to illustrate the vortex deformation or erosion. That is why a single particle is seeded at each vortex center initially; it helps follow each vortex through time.

Particles are also seeded initially along two circles in the western vortex. The circles have radii $0.4 R$ ( 5 particles along this circle), and $0.8 R$ (with 8 particles). The aim of these "inner and outer rings of particles" is to confirm how eroded the vortex becomes, with time. The peripheral particles, if torn away from the vortex, indicate that the peripheral layers of the vortex are stripped off.

Particles are seeded at the bottom and at the top of the topographic slope to show the cross-slope transfers of fluid.

The trajectory of the central particles (particles initially at each vortex center) is shown in figure 10a. Both particles drift upslope and eastward via the interaction of each vortex with the topographic wave; over the slope, the particles are close to each other. Over the shallower part of the domain, the loops in these particle trajectories 


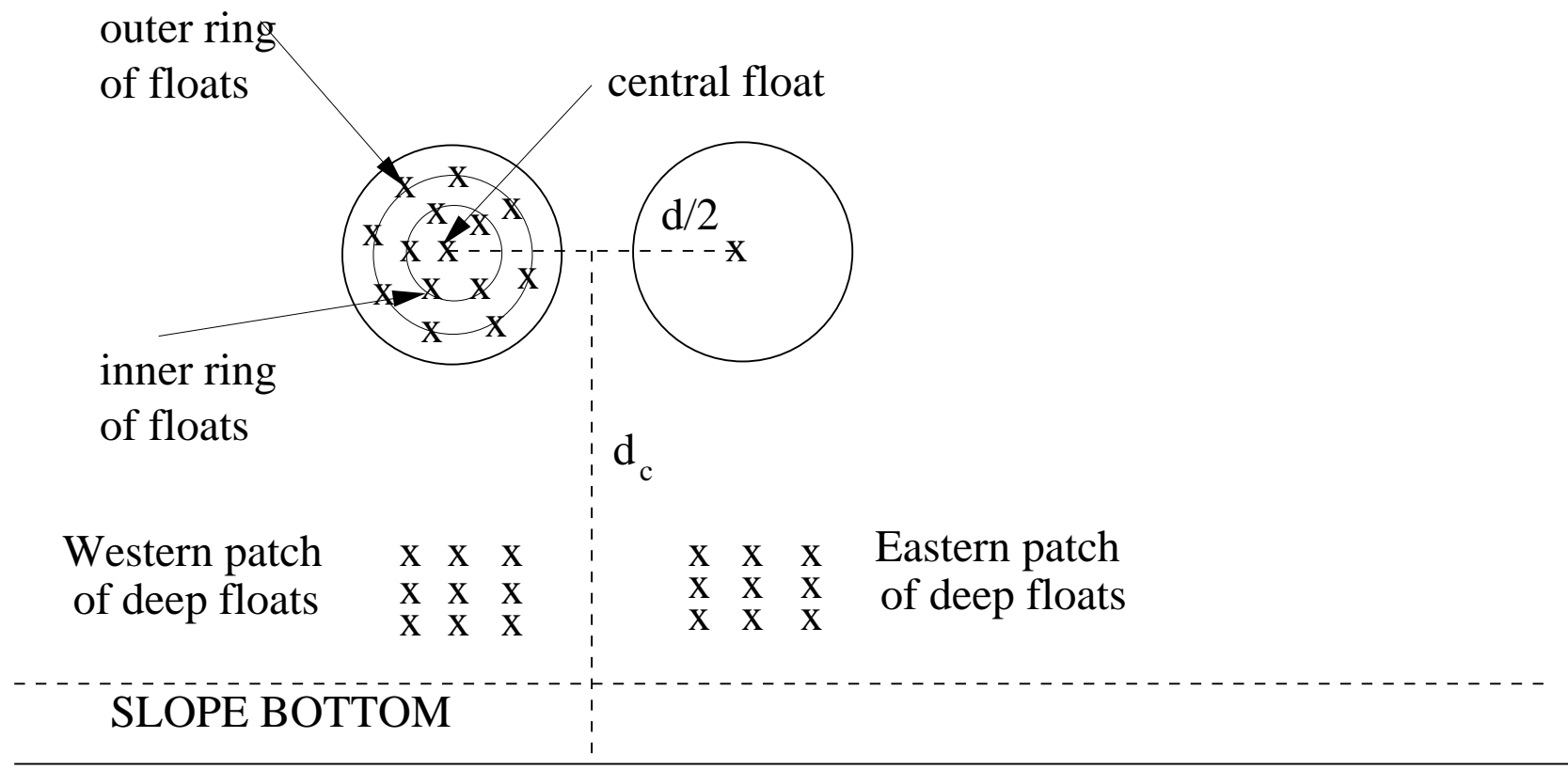

\section{MID SLOPE}

\section{SLOPE TOP}

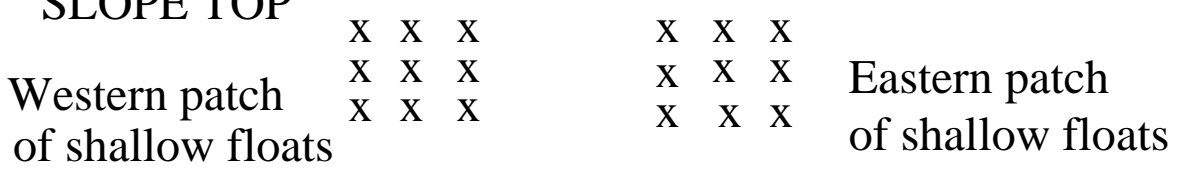

Figure 9: General sketch of the position of the various sets of individual particles numerically seeded in the model. 
a)

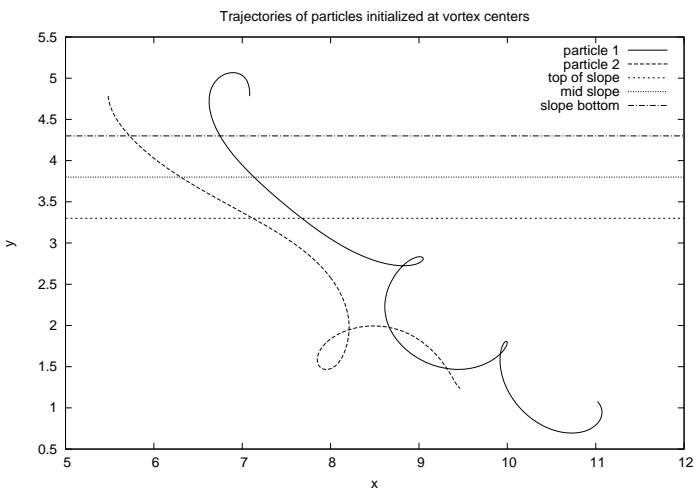

c)

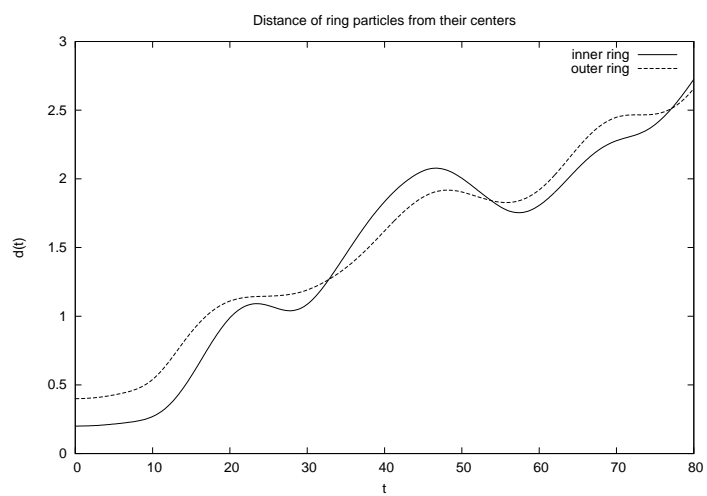

d)

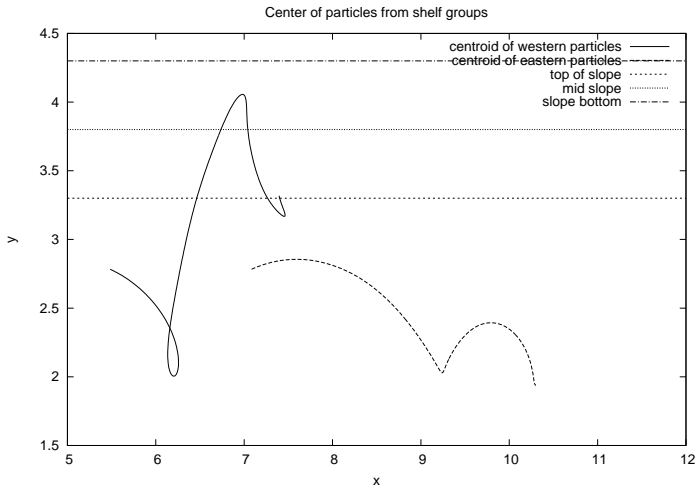

b)

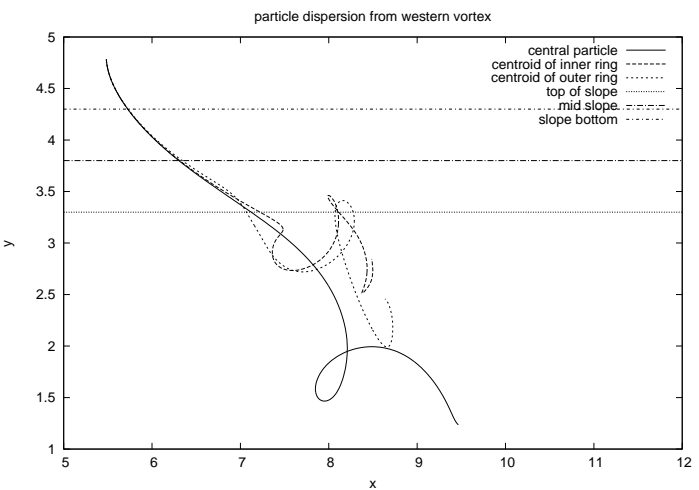

e)

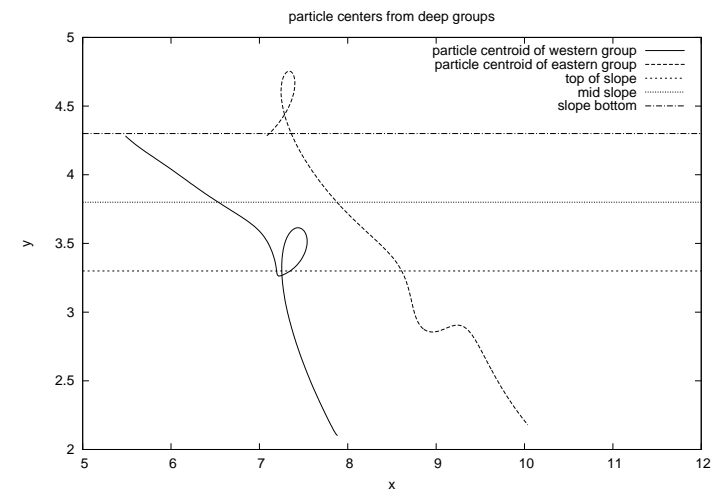

Figure 10: (a) Trajectories of the particles initially located at the vortex centers ; (b) trajectories of the centroids of particles seeded along circles in the western vortex ; (c) distance between the particles and their centroids in each vortex; (d) trajectories of particle centroids from the patch at the top of the slope; (e) trajectories of particle centroids from the patch at the bottom of the slope. 
indicate the tumbling of the two vortices during their interaction. Finally the two particles lie at a distance comparable to their initial separation. This is due to the straining out of the two vortices in the second part of their evolution. The particle from the easternmost vortex lies in the final vortex, while the particle from the westernmost vortex lies out of this final vortex, to the Northwest.

Secondly, the evolution of rings of particles is studied. Figure 10p indicates that, over the upper slope, the outer ring of particles diverges from the central particle. The deformation reaches the vortex core shortly after. Then the two rings of particles have - on average - similar trajectories. Only late in the evolution, do the trajectories of the outer and inner ring centroids slowly diverge.

The (root-mean squared) average distance of the rings of particles from their centroids is then plotted versus time (figure 10 c). It provides a qualitative illustration of the vortex evolution. Deformation increases as the westernmost cyclone crosses the slope uphill (between $t=10$ and $t=20$ ). This leads to the filamentation of this vortex, once in the shallow domain. The filament thus created is elongated while advected in the clockwise direction by the merged vortex. This advection pushes the filament downslope. This corresponds to the second increase in particle dispersion (between $t=35$ and $t=45)$. The tip of this filament rolls up as a small vortex over the lower slope; then this small vortex moves upslope, is elongated and finally moves downslope. Simultaneously, the merged vortex is elongated and splits over the upper slope. These two effects correspond to the last increase in particle dispersion $(t=60-80)$.

In figure $10 \mathrm{~d}$, the particle centroids are shown starting from the top of the slope. Both the particles east and west of the cyclones loop and move into the shallow region. Particles seeded at the bottom of the slope climb upslope, confirming the previous results and conclusions (see figure [10?).

For the case $d_{c} / R=1, d / R=3.8$, particles are seeded similarly in the domain. The particles initially at the vortex centers drift upslope and follow parallel trajectories before diverging; they do not come close together (figure not shown). Similarly, all particles in the western vortex follow comparable trajectories. Only the outer particles slowly diverge in the final stage of the simulation. 
a)
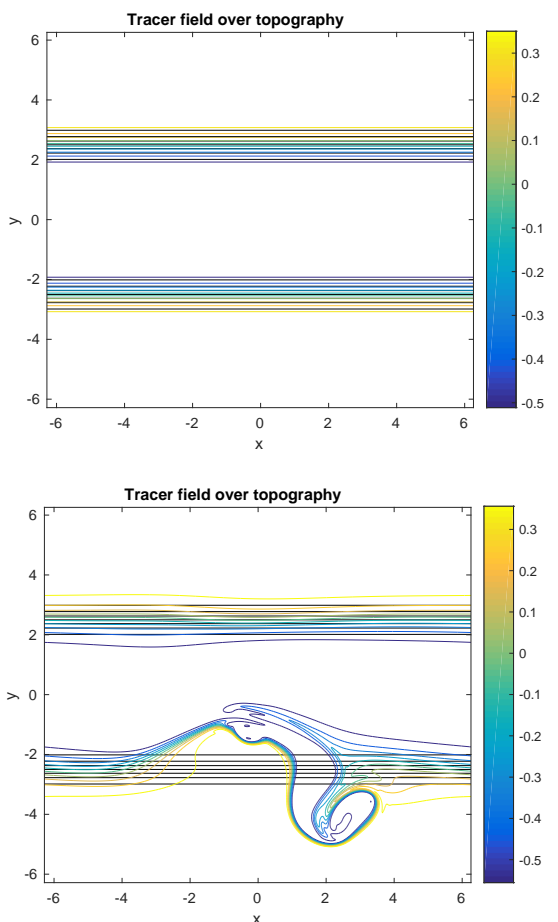

b)
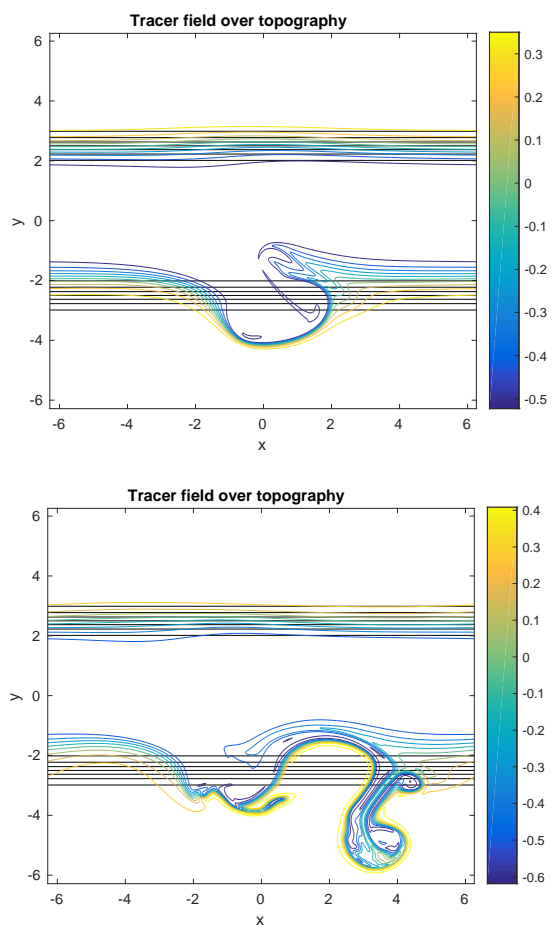

d)

Figure 11: Evolution of maps of tracer field with time; a) $t=0, \mathrm{~b}) t=18$, c) $t=39$, d) $t=60$ (subplots a,b,c,d). The horizontal black lines are isobaths (indicating the bottom slope), and the color scale characterizes the intensity of the tracer concentration.

\subsubsection{Passive tracer evolution}

To investigate further the cross-slope exchange, we add a passive tracer to the flow. This initial tracer distribution is continuous spatially, and is specified in section 2 . It is higher in the shallow region and lower in the deep region.

We present the horizontal maps of tracer in the case $d / R=3.2, d_{c} / R=2$ in figure 11 .

The tracer field is rapidly advected into the shallow region, and deformed by the twovortex flow; it is also advected into the deep region. The tracer gradients substantially increase. The tracer isolines correspond to the vorticity isolines and in particular those of the filaments; this can be seen in the final map of tracer and of vorticity, see figure 
a)

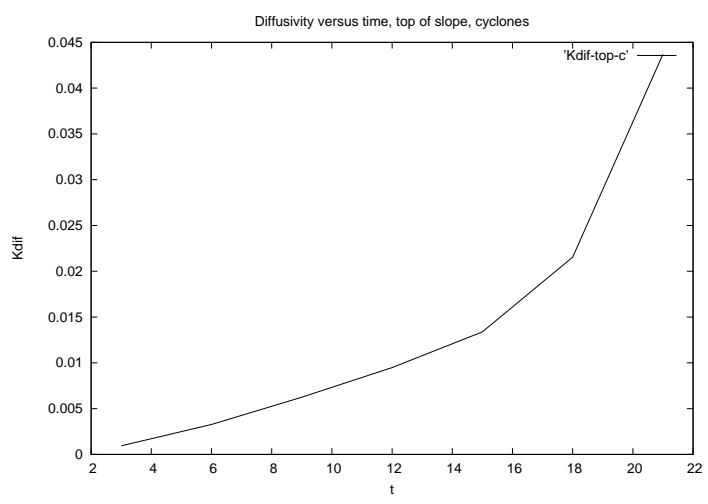

b)

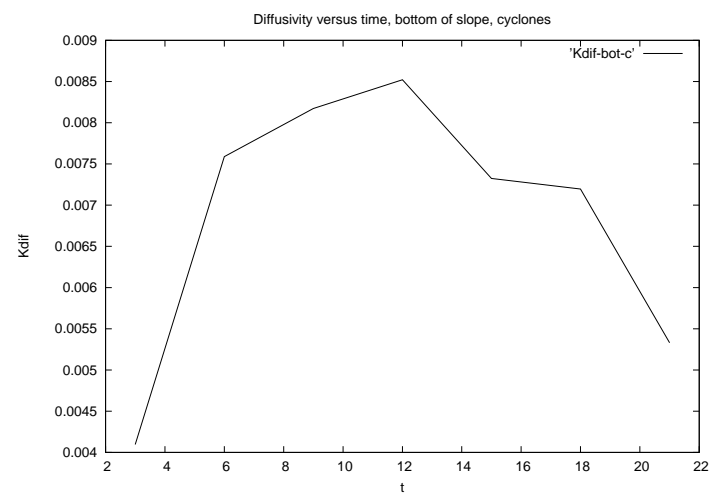

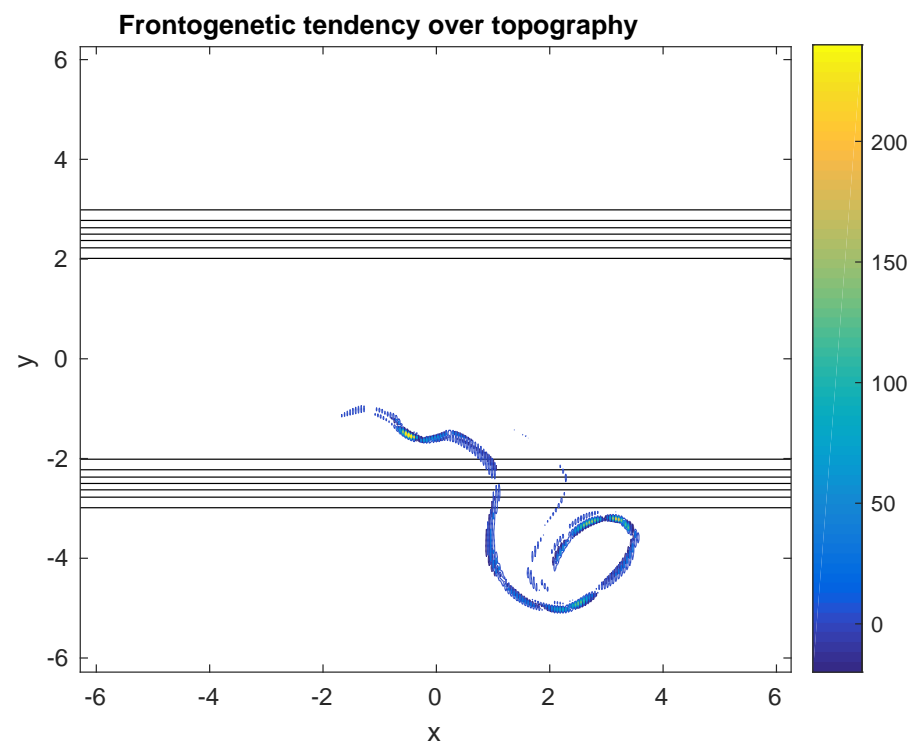

C

Figure 12: Early time evolution of the tracer diffusivity, (a) at the upper slope, averaged between $y_{t}+L_{t} / 2$ and $y_{t}+L_{t}$; (b) at the lower slope and over flat bottom, averaged between $y_{t}-L_{t}$ and $y=0$. The frontogenetic tendency is plotted in (c) at $t=39$. The black lines are isobaths (indicating the bottom slope), and the color scale characterizes the intensity of the frontogenetic tendency. 
11d.

The diffusivity, computed as indicated in appendix 2, shows a linear increase ("ballistic regime") for early times, over the upper slope, see figure 12 a. At the lower slope, and near the center of the domain, diffusion increases linearly at short times, then decreases and becomes weaker, see figure $12 \mathrm{~b}$.

In the ocean, the corresponding diffusivities would be $K_{t o p}=4050 \mathrm{~m}^{2} / \mathrm{s}$ and $K_{b o t}=$ $765 \mathrm{~m}^{2} / \mathrm{s}$. The first value is characteristic of a turbulent horizontal mixing process in the western boundary currents (this diffusivity is called "suppressed eddy diffusivity" by Klocker and Abernathey, 2014); on the contrary, the latter value is more characteristic of horizontal diffusivity in the center of a mid-latitude gyre.

The frontogenetic tendency (see appendix 3 and figure 12c) confirms the rapid increase of the tracer gradients in the vicinity of the vortex pair at the upper slope.

\subsection{Influence of the orientation of the vortex pair}

When the two cyclones initially lie perpendicularly to the slope, the vortex closest to the slope is advected to the southeast by the topographic wave and by the secondary poles. The distance between the two primary vortices then increases to the point that no merger occurs (see figure 13). Therefore, the initial orientation of the vortices has a major effect on the outcome of their interaction.

\subsection{Influence of the vortex polarity: evolution of two anticy- clones}

We now focus on the interaction between two anticyclones, near a topographic slope. Figure 14 presents the nonlinear regime distribution in the $\left(d / R, d_{c} / R\right)$ plane.

Clearly, merger occurs much more easily than over flat bottom:

- symmetric merger has a critical distance $d=3.3 R$ when the vortices lie at 6 radii from the slope, but this distance increases to $d=3.9 R$ at 5 radii from the slope.

- asymmetric merger occurs for two anticyclones lying between 2 and 4 radii away from the slope; in this case, the critical merger distance is beyond $6 R$. Only when the two anticyclones are very close to the slope, does the topographic wave separate them even if they lie close to each other initially $(d / R=3.2)$. 


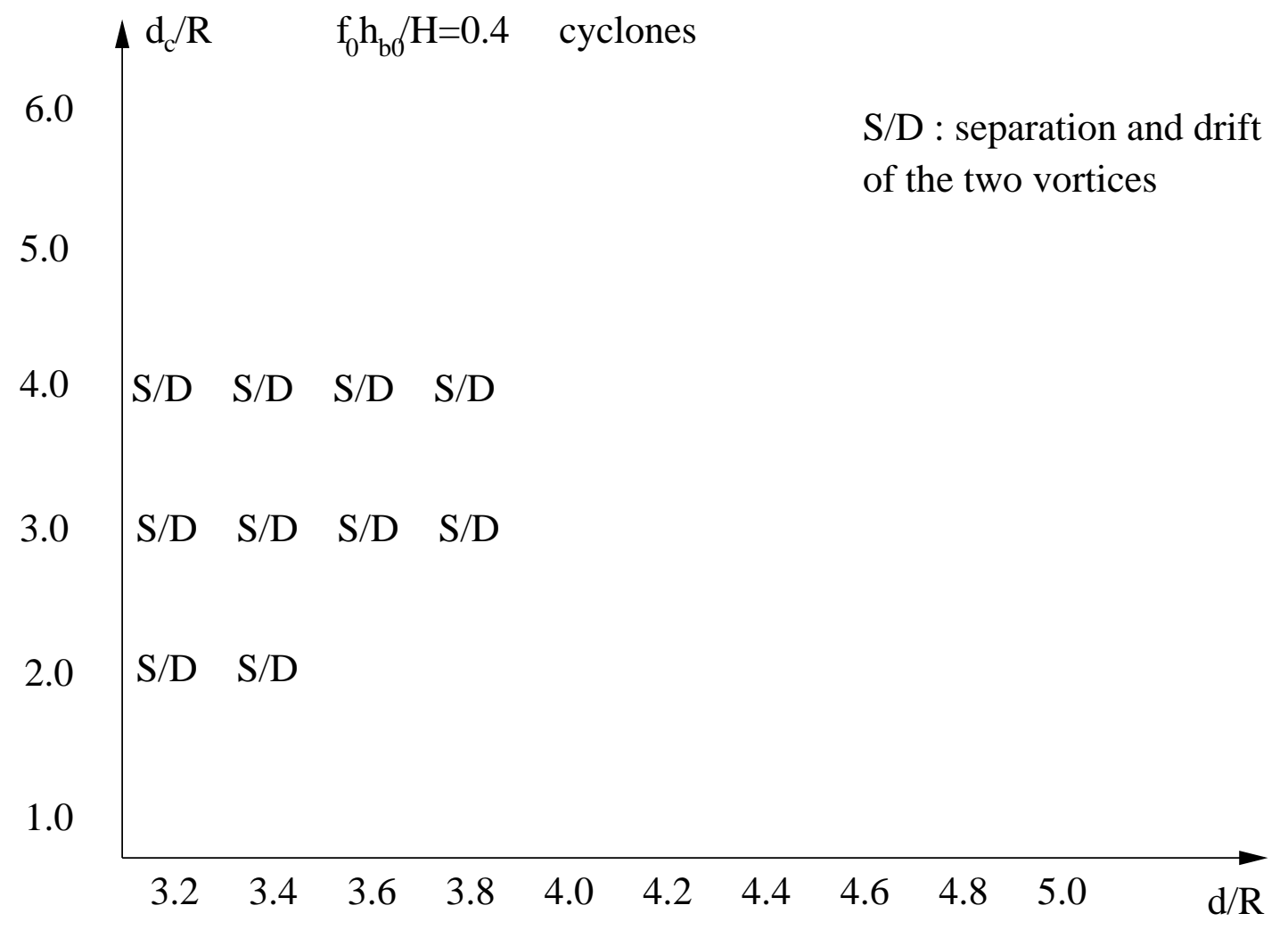

Figure 13: Nonlinear regimes of two cyclones when they lie initially perpendicularly to the slope. 


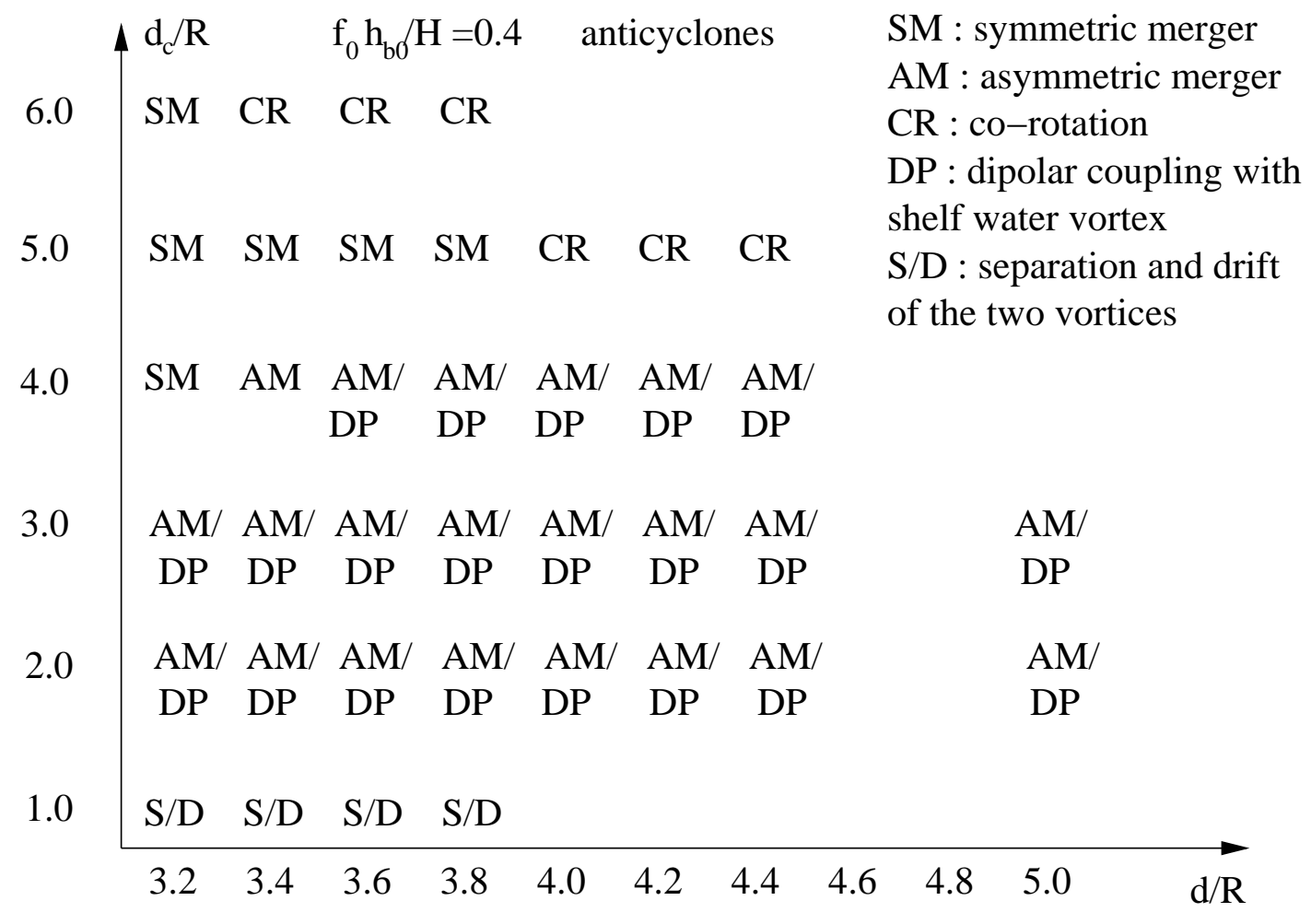

Figure 14: Nonlinear regimes of two anticyclones lying initially parallel to the slope. 
a)

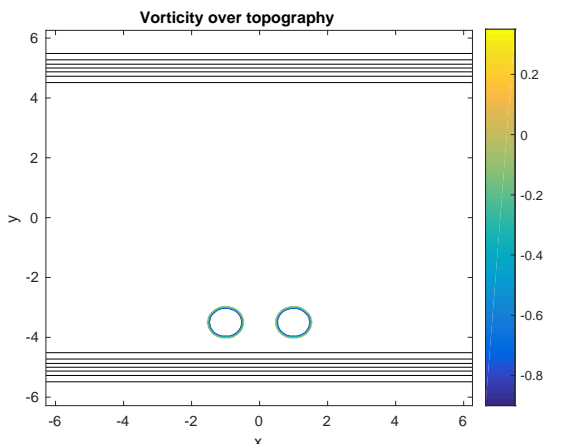

c)

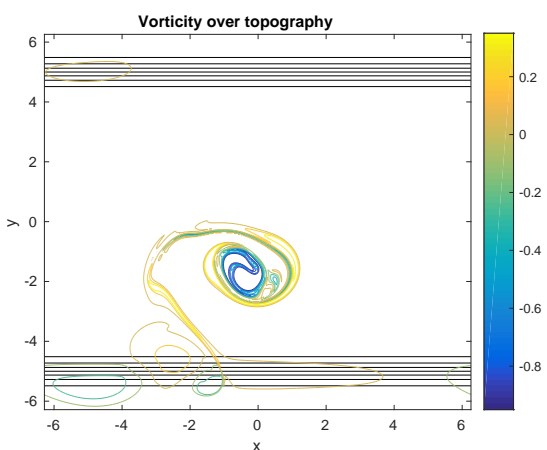

b)
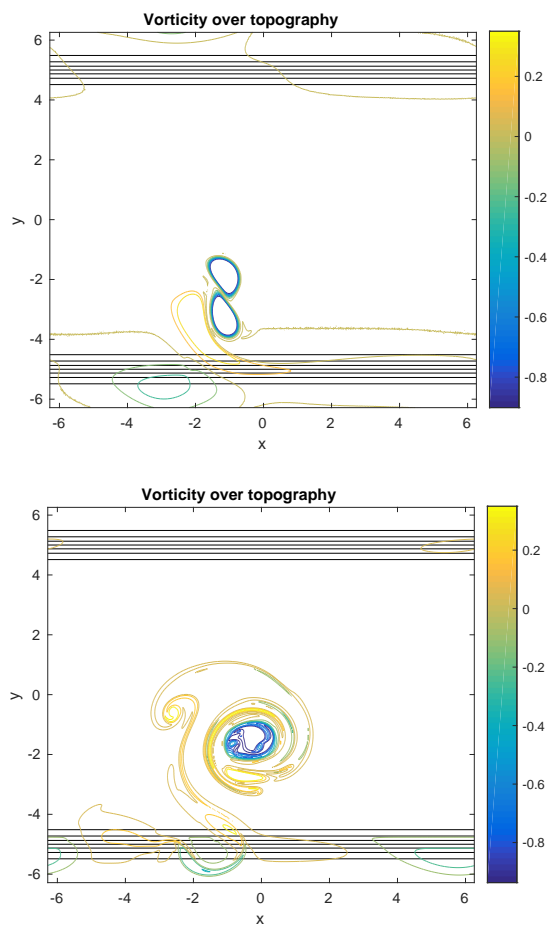

d)

Figure 15: Time series of vorticity maps for the evolution of two anticyclones, initially parallel to the slope, with $f_{0} h_{b 0} / H=0.4$, and $d / R=4, d_{c} / R=3$. Times shown are $t=0,18,36,54$ (subplots a, b,c,d). The black lines are isobaths (indicating the bottom slope), and the color scale characterizes the intensity of the relative vorticity.

The mechanism favoring merger is the formation of a positive (secondary) vorticity pole, which detaches from the slope into the deep domain and couples with the anticyclone closest to the slope (see figure [15a for the case $d / R=4, d_{c} / R=3$ ). This dipole propagates northwestward and hits the second anticyclone (see figure 15b), thus achieving merger from large distances. In the last stage, peripheral cyclones, generated by the roll-up of filaments, lie around the merged vortex.

The topographic waves generated on the slope are analyzed during the early evolution of the flow, and their modal decomposition is shown. At the upper slope, only the distant flow field of the vortices is felt and the longest wave dominates (figure 16a). At the lower slope, the vortex flow influences the modal contents and a shorter wave $(k=2)$ is also noticeable (figure $16 \mathrm{~b})$. The fact that shorter waves are weaker during the interaction of two anticyclones (compared to that for two cyclones), is due to the 
a)

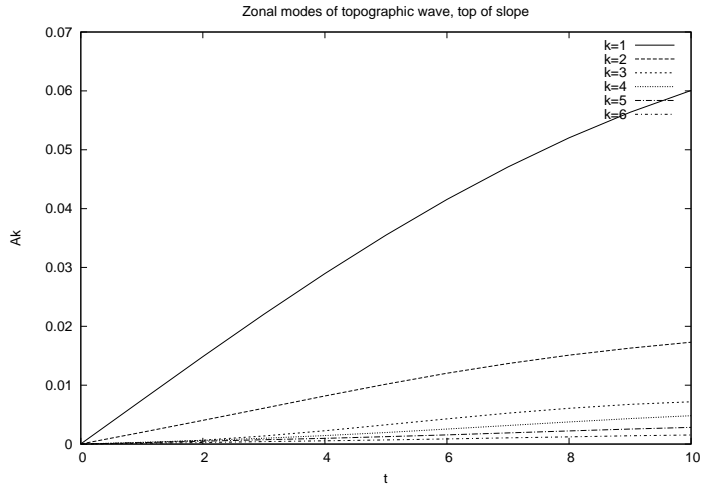

b)

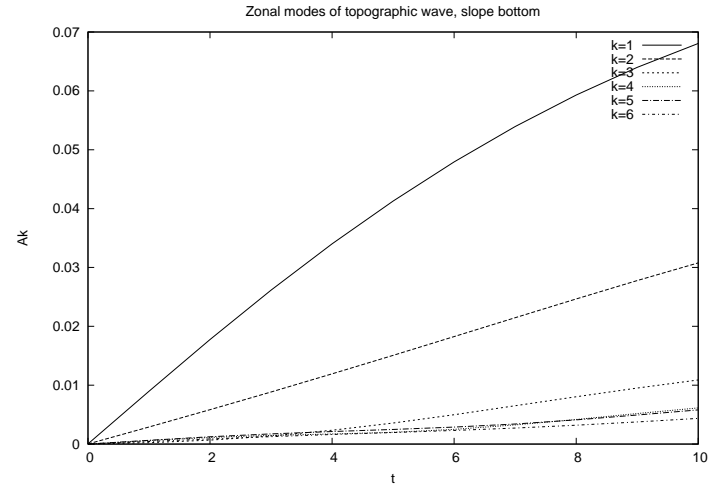

Figure 16: Time series of topographic wave amplitude for the various zonal modes from $k=1$ to $k=6$ at the upper slope (a) and at the lower slope (b), for the interaction of two anticyclones.

drift of the two anticyclones away from the slope. The farther away from the slope the vortices are, the more dominant long waves become in the topographic wave.

Finally, we studied the tracer evolution in this case. The diffusivity also follows a linear increase ("ballistic regime") at early times (see figure 17), but rapidly stabilizes at the upper slope.

In the ocean, the corresponding diffusivities would be $K_{\text {top }}=540 \mathrm{~m}^{2} / \mathrm{s}$ and $K_{\text {bot }}=$ $580 \mathrm{~m}^{2} / \mathrm{s}$, characteristic of weak diffusion. The diffusivity is weaker here than for two cyclones, because the anticyclones drift away from the shallow region; only one patch of positive vorticity is extracted from this shallow region. The secondary poles of vorticity (filaments) then evolve in a region of uniform tracer concentration, away from the slope. To sum up, the interaction of two anticyclones near the topographic slope facilitates their merger, but leads to vortex filamentation which reduces the merger efficiency.

\section{$5 \quad$ Influence of step height}

Here we set the topographic vorticity to $f_{0} h_{b 0} / H=0.8$.

Several differences with the case of shallower bathymetry, are observed (see figure 18): 1) For cyclones, merger occurs for larger initial distances over the taller topography, but the efficiency of the merging process is reduced; more filamentation occurs on the slope, often leading to the complete destruction of the westernmost vortex, or of both 
a)

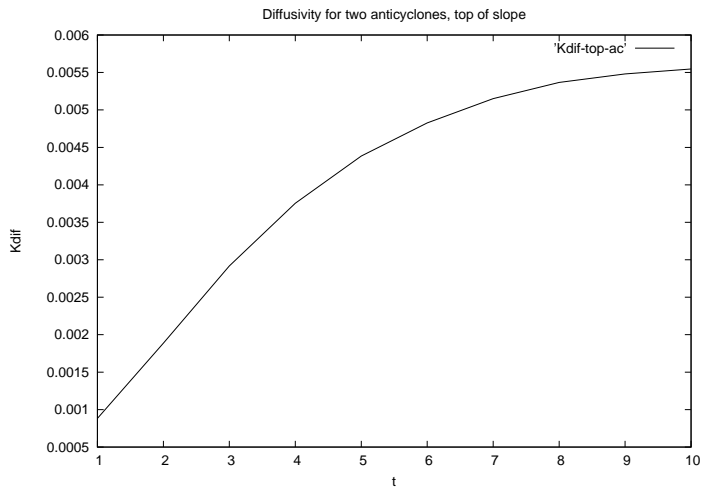

b)

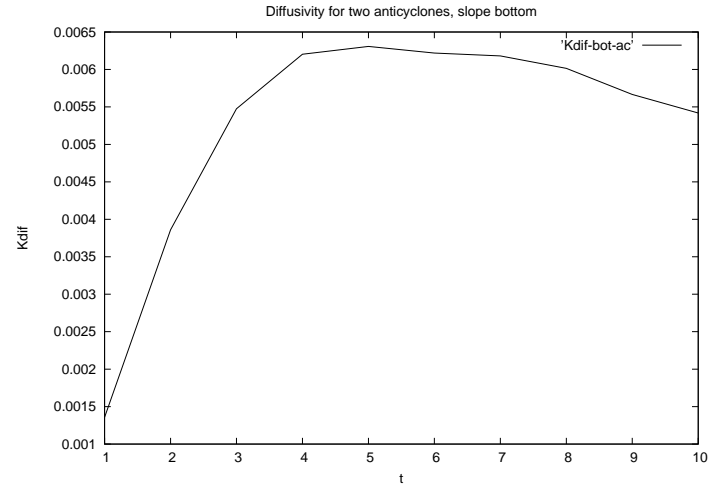

Figure 17: Early time evolution of the tracer diffusivity, (a) at the upper slope; (b) at the lower slope, for the interaction of two anticyclones.

vortices.

2) In that respect, for cyclones close to the slope (but not on the slope), the critical merger distance increases to $d / R=5.3$ instead of $d / R=4.4$.

3) For anticyclones, which drift away from the slope, merger is favored by the topographic effect, for the range of topographic heights considered here : a topographic (secondary) cyclone pairs with the easternmost (primary) anticyclone and advects it towards the other anticyclone.

4) For $d_{c} \sim 2 R$, the critical merger distance is larger than for the shallower topography. 5) Only when the two anticyclones lie very near the slope, do they merge more easily when topography is tall. This is related to the stronger dipolar effect favoring this merger.

To quantify the stronger shearing effect of tall topographies, a simple model is proposed in appendix 4.

\section{Conclusions}

To start investigating vortex interaction near a continental slope in the ocean, we have used a very simple model and configuration. They have limitations, which will be listed below. Nonetheless, they provided interesting information on the possible merger of two like-signed vortices near a topographic step (or slope): 


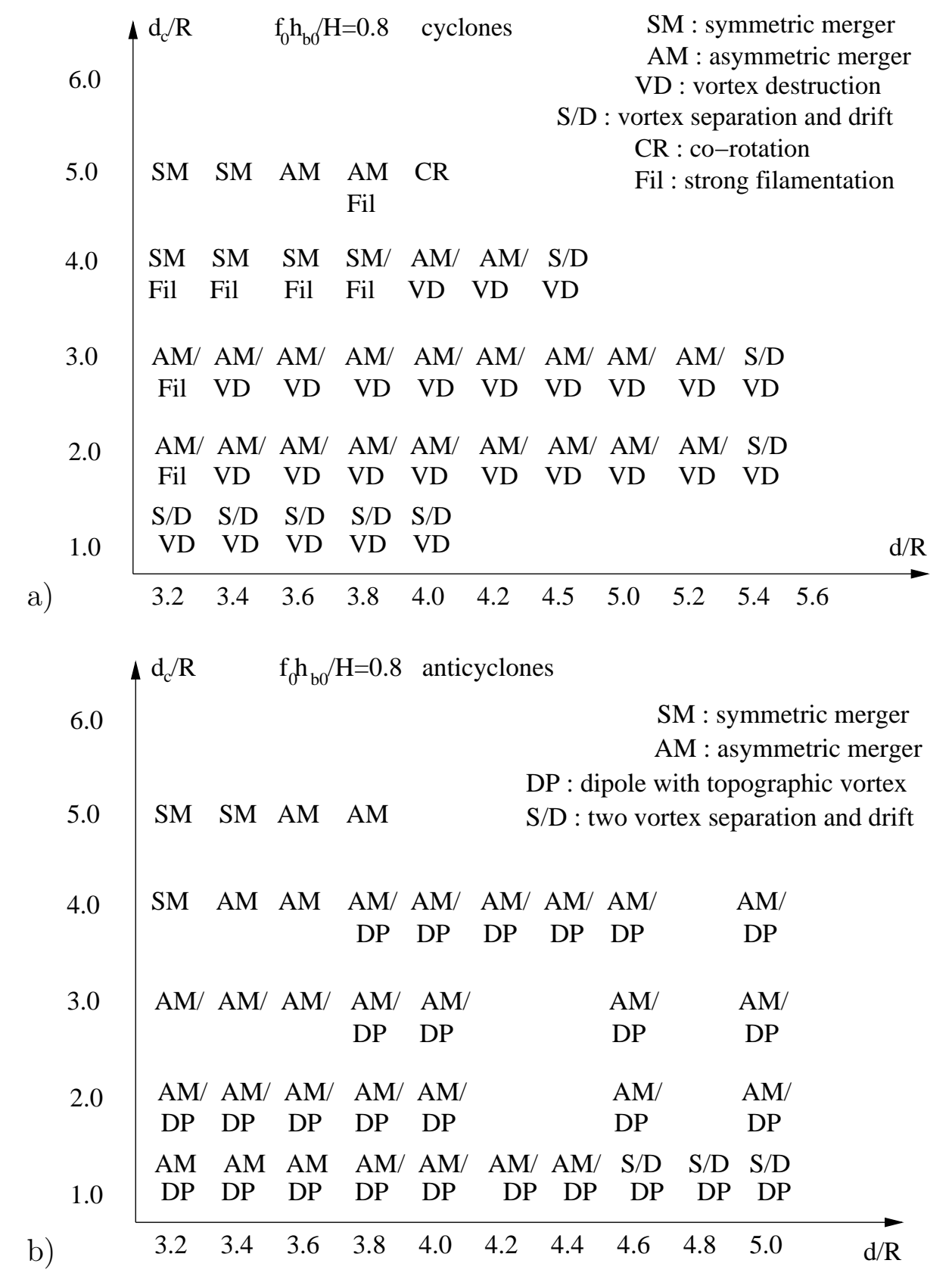

Figure 18: Nonlinear regimes of two cyclones (a) or of two anticyclones (b), initially parallel to the topographic slope, with respect to their initial separation (scaled by their radius), $d / R$, and to their initial distance to the slope $d_{c} / R$. The topographic height, in terms of vorticity, is now $f_{0} h_{b 0} / H=0.8$. The various regimes are indicated in the figure. 
- two cyclones merge more easily near a topographic slope but the merging efficiency is reduced due to filamentation and wave dispersion; the critical distance for merger can then substantially increase (to more that 5.5 radii).

- long topographic waves grow first on the slope, followed by shorter modal components (which are stronger close to the vortices);

- a simple model is derived to anticipate these effects in the long range limit (linear approximation);

- both the topographic wave and the secondary vortices tend to erode cyclones climbing upslope, favoring diffusion of tracer.

- As a qualitative illustration of these processes and evolutions, particles seeded in the cyclone cores also indicates the progressive erosion of the cyclones as they climb upslope; particles, seeded at the upper and lower slope, follow the global upward motion.

- Tracer diffusion confirms this tendency for cyclones and diffusivities were calculated, showing a ballistic regime initially. Diffusivity is larger in the shallow region and during the interaction of two cyclones, close to the slope (and close to the tracer gradient). This is due to the strong shearing and straining effects of the secondary vorticity patches. This effect is less important for two anticyclones, which drift away from the slope into the deep, open domain: the intensity and spatial concentration of secondary vorticity is then weaker and it is located in a region of uniform tracer concentration.

- The initial orientation of the two vortices with respect to the slope is important: if the two cyclones lie initially perpendicularly to the slope, they are separated and do not merge.

- On the contrary, two anticyclones easily merge near a topographic slope, because one is ejected towards the other via coupling with secondary (topographic) vortices.

- In many instances, both for cyclones and for anticyclones, vortex merger is favored, the critical merger distance is increased, but the efficiency of the merging process is reduced.

- Finally, for taller topographies, the critical merger distance is again increased and merger efficiency decreased; the slope can scatter or erode completely one vortex; anticyclone merger near the shelf is possible.

For application to the ocean, this study should be extended to very tall topographies using first the homogeneous shallow-water model. Then, two cases can occur: either 
the shelf is wide and the present study is a starting point, or the shelf is very narrow, and vortex merger near a vertical wall is a preferable starting point.

In both cases, to attain realism for oceanic situation, the model should include more dynamical elements: density stratification, a variable Coriolis parameter (planetary beta effect), and possibly more complex topography (canyons, seamounts) and local currents. Preliminary simulations of two anticyclones near a coast, on the beta plane, indicate that they merge without drifting offshore, being maintained against the coast by beta effect. This merger forms a vortex not larger than any of the initial two, since Rossby waves disperse energy during the process. The addition of a coastal current to this configuration was also tested; when the vortices interact with this current, a saddle point is can be created which is critical for cross-shore fluid transfer (Capet and Carton, 2004; Vandermeirsch et al., 2003).

As a first step towards realism, this study will be generalized to a stratified fluid in a forthcoming paper.

\section{Acknowledgements}

This work is a contribution to the PHYSINDIEN research program. It was supported by CNRS-RFBR contract PRC 1069/16-55-150001. The authors thank Dr David Griffin (CSIRO, Australia) for his authorisation to use figure 1.

\section{Appendix 1 : point vortex pair, topographic vorticity front in- teraction}

Here, we compute the effect of two point vortices on a topographic vorticity front in the long distance limit. We assume here that the topography is a step with a vorticity jump $\delta q=-f_{0} h_{b 0} / H$ northward. The configuration is the same as in figure 9, but now, the two vortices have zero radius and finite circulation $\Gamma$. It is also assumed that their

mutual distance $d$ is very small compared with their distance $d_{c}$ to the topographic slope, thus justifying the long distance limit.

In that respect, we can use a dipolar expansion of the streamfunction induced by the 
point dipole and truncate it at lowest order, or, similarly, apply the Gauss theorem to the vorticity distribution. Thus, the far field of the streamfunction, at the topographic step, is

$$
\psi(x, y=0)=\frac{\Gamma}{2 \pi} \log \left[x^{2}+d_{c}^{2}\right]
$$

We can now follow the theory of vortex - potential vorticity front interaction developed in Stern and Flierl (1987), in the particular case where there is no basic zonal flow. Using the linear theory (for large distances between the point dipole and the front), the potential vorticity interface deviation from its initial position $y=0$ is

$$
\eta(x)=\frac{\Gamma d_{c}}{\pi \delta q\left(x^{2}+d_{c}^{2}\right)}
$$

(one can note that, in fact, the interface would be initially at $y=-y_{t}$ in the numerical model.)

Again, as in the case dealt with by Stern and Flierl (1987), the meander has a circulation equal and opposite to that of the point dipole; the meridional velocity at $y=0$ is

$$
v(x)=\frac{\Gamma x}{2 \pi\left(x^{2}+d_{c}^{2}\right)}
$$

The Fourier transform of the interface deviation $\eta$ is $\Gamma d_{c} \exp \left(-k d_{c}\right) /(\delta q)$.

\section{Appendix 2 : Diffusive evolution of a tracer from an initial front}

Our objective here is to provide a simple estimate of the diffusivity of a continuous tracer across the topographic slope. To do so, we start from the $2 \mathrm{D}$ diffusion equation

$$
\partial_{t} T=K\left[\partial_{x}^{2}+\partial_{y}^{2}\right] T, \quad(x, y) \in[-L / 2, L / 2] \times[-L / 2, L / 2], t>0
$$

where $T(x, y, t)$ is the tracer concentration. We integrate this equation between two isolines of $y$ (e.g. the upper and lower boundaries of the topographic slope) and over the length of the domain in $x$. We use the periodicity of the domain to eliminate the 
integrated term $\left[\partial_{x} T\right]_{-L / 2}^{L / 2}$ and we obtain:

$$
\frac{d}{d t} \int_{0}^{L} d x \int_{y_{1}}^{y_{2}} T d y=K \int_{0}^{L} d x\left[\partial_{y} T\right]_{y_{1}}^{y_{2}}
$$

This equation is the budget of tracer in the region thus determined. By calculating the time derivative in the left-hand side, and the integral in the right-hand side, we obtain the diffusivity $K$.

\section{Appendix 3: the frontogenetic tendency}

Consider a passive tracer $T$, conserved during its advection by the flow

$$
\frac{d T}{d t}=\partial_{t} T+J(\phi, T)=0
$$

Then, the gradient of the tracer obeys the equation

$$
\frac{d \vec{\nabla} T}{d t}=-\vec{\nabla} \vec{u} \cdot \vec{\nabla} T
$$

and its norm varies as

$$
\frac{d|\vec{\nabla} T|^{2}}{d t}=-(\vec{\nabla} T)^{T} \vec{\nabla} \vec{u} \cdot \vec{\nabla} T
$$

The right hand side of this equation is called the frontogenetic tendency and is written

$$
F(u, v, T)=-\left[\partial_{x} u\left(\partial_{x} T\right)^{2}+\left(\partial_{x} v+\partial_{y} u\right) \partial_{x} T \partial_{y} T+\partial_{y} v\left(\partial_{y} T\right)^{2}\right]
$$

It indicates where the tracer gradients increase or decrease.

\section{Appendix 4: Shearing and dispersive effects of topography}

Using the theory above (appendix 1), we know that (in the linear regime) the secondary vortices, which result from the occlusion of the topographic wave meanders, have about the same circulation $\Gamma=\pi R^{2} \omega$ as the primary vortices. If they lie on both 
sides of the slope, the maximum shear that they create is $\frac{d U}{d y}=\frac{\Gamma}{2 \pi \times 4 L_{t}^{2}}$.

It is also known that, if $(d U / d y) / \omega>0.15$, the primary vortices will be sheared out (McWilliams, 1991). In the present case, this ratio is 0.125 which explains the substantial shearing out of the cyclone pairs near the slope (for $d_{c} \leq 4 R$ ).

The topographic beta effect is $\beta_{T}=\frac{f_{0}}{H} \frac{d h_{b}}{d x}$. It acts on the primary vortices which have maximal azimuthal velocity $V_{m}=\omega R / 2$. The Rhines scale separating the vortical and wave regimes is $L_{R}=\sqrt{V_{m} / \beta_{T}}$.

For the shallowest topography, $L_{R}=R$ and for the tallest, $L_{R}=R / \sqrt{2}$. Since the Rhines scale is comparable with, or smaller than, the vortex radius, topography has a strong dispersive effect on cyclones as they move upslope.

\section{$7 \quad$ References}

Aguiar A.C.B., Peliz A. and X. Carton, 2013: A census of meddies in a long-term high-resolution simulation. Progress in Oceanography, 116, 80-94.

Angot P., Bruneau C.H. and P. Fabrie, 1999: A penalisation method to take into account obstacles in viscous flows. Num. Math., 81, 497-520.

Bambrey R.R., Reinaud J.N. and D.G. Dritschel, 2007: Strong interactions between two co-rotating quasi-geostrophic vortices. J. Fluid Mech., 592, 117-133.

Bertrand, C. and X.J. Carton, 1993: Vortex merger on the beta-plane. Compte-Rendus Acad. Sc. Paris, 316(II), 1201-1206.

Capet X. and X. Carton, 2004: Nonlinear regimes of baroclinic boundary currents. $J$. Phys. Oceanogr., 34, 1400-1409.

Carnevale G.F., Cavazza, P., Orlandi, P. and R. Purini, 1991: An explanation for anomalous vortex merger in rotating tank experiments. Phys. Fluids A, 3(5), 14111415.

Carton X.J., 1992: The merger of homostrophic shielded vortices. Europhys. Lett., 18, 8, 697-703.

Carton X., Daniault N., Alves J., Chérubin L. and I. Ambar, 2010: Meddy dynamics and interaction with neighboring eddies southwest of Portugal : observations and modeling. J. Geophys. Res., 115, article C06017, doi:10.1029/2009JC005646, 23 pp. Carton X., Le Cann B., Serpette A. and J. Dubert, 2013: Interactions of surface and 
deep anticyclones in the Bay of Biscay. J. Mar. Syst., 109-110, S 45 - S 59.

Cenedese C., Todd R.E., Gawarkiewicz G.G., Brechner Owens W. and A.Y. Shcherbina, 2013: Offshore transport of shelf waters through interaction of vorties with a shelfbreak current. J. Phys. Oceanogr., 43, 905-919.

Dewar W.K., Berloff P. and A.M. Hogg, 2011: Submesoscale generation by boundaries. J. Mar. Res., 69, 4-6, 501-522.

Dritschel, D.G., 1985: The stability and energetics of corotating uniform vortices. J. Fluid Mech., 157, 95-134.

Dritschel, D.G., 1986: The nonlinear evolution or rotating configurations of uniform vorticity. J. Fluid Mech. 1986, 172, 157-182.

Dritschel D.G. 2002: Vortex merger in rotating stratified flows. J. Fluid Mech., 444, 83-101.

Dunn D.C., McDonald N.R. and E.R. Johnson, 2001: The motion of a singular vortex near an escarpment. J. Fluid Mech., 448, 335-365.

Griffiths R.W. and E.J. Hopfinger, 1987: Coalescing of geostrophic vortices. J. Fluid Mech., 178, 73-97.

Hardenberg J. von, McWilliams, J.C., Provenzale, A., Shchepetkin, A. and J.B. Weiss, 2000: Vortex merging in quasi-geostrophic flows. J. Fluid Mech., 412, 331-353.

Hinds A.K., Johsons E.R. and N.R. McDonald, 2007: Vortex scattering by step topography. J. Fluid Mech., 571, 495-505.

Klocker A. and R. Abernathey, 2014: Global patterns of mesoscale eddy properties and diffusivities. J. Phys. Oceanogr., 44, 3, 1030-1046.

L'Hegaret P., Carton X., Ambar I., Menesguen C., Hua B.L., Chérubin L., Aguiar A., Le Cann B., Daniault N. and N. Serra, 2014: Evidence of Mediterranean Water dipole collision in the Gulf of Cadiz. J. Geophys. Res., 119, 8, 5337-5359.

L'Hegaret P., Duarte R., Carton X., Vic C., Ciani D., Baraille R. and S. Correard, 2015: Seasonal mesoscale variability in the Arabian Sea from HYCOM model and observations: impact on the Persian Gulf Water path. Ocean Science, 11, 667-693.

McDonald N.R., 1998: The motion of an intense vortex near topography. J. Fluid Mech., 367, 359-377.

McWilliams J.C. 1991: Geostrophic Vortices. In: Nonlinear Topics in Ocean Physics: Proceedings of the International School of Physics Enrico Fermi , Course 109, A.R. Osborne, ed., North-Holland, Elsevier Science Publishers B.V., Amsterdam, 5-50. 
Melander M.V., Zabusky N.J. and J.C. McWilliams, J.C., 1987: Asymmetric vortex merger in two dimension: Which vortex is "victorious" ? Phys. Fluids A, 30(9), 26102612 .

Melander M.V., Zabusky N.J. and J.C. McWilliams, 1988: Symmetric vortex merger in two dimensions: causes and conditions. J. Fluid Mech., 195, 303-340.

Meleshko V.V., 1994: Nonstirring of an inviscid fluid by a point vortex in a rectangle. Phys. Fluids, 6, 6, DOI:10.1063/1.868047.

Meunier, P., Ehrenstein, U., Leweke, T. and M. Rossi, 2002: A merging criterion for two-dimensional co-rotating vortices. Phys. Fluids, 14(8), 2757-2766.

Molemaker M.J., McWilliams J.C. and W.K. Dewar, 2015: Submesoscale instability and generation of mesoscale anticyclone near a separation of the California Undercurrent. J. Phys. Oceanogr., 45, 613-629.

Oey L.Y. and H.C. Zhang, 2004: The generation of subsurface cyclones and jets through eddy-slope interaction. Cont. Shelf Res., 24, 18, 2109-2131.

Overman, II, E.A. and Zabusky, N.J., 1982: Evolution and merger of isolated vortex structures. Phys. Fluids, 25(8), 1297-1305.

Ozugurlu, E., Reinaud, J.N. and D.G. Dritschel, 2008: Interaction between two quasigeostrophic vortices of unequal potential-vorticity. J. Fluid Mech., 597, 395-414.

Pavia E.G. and B. Cushman-Roisin, 1990: Merging of frontal eddies. J. Phys. Oceanogr., 20, 1886-1906.

Reinaud, J.N. and D.G. Dritschel, 2002: The merger of vertically offset quasi-geostrophic vortices. J. Fluid Mech., 469, 297-315.

Reinaud, J.N. and D.G. Dritschel, 2005: The critical merger distance between two corotating quasi-geostrophic vortices. J. Fluid Mech., 522, 357-381.

Roenby J. and H. Aref, 2010: Chaos in body-vortex interactions. Proc. R. Soc. A, 472, 2187, doi:10.1098/rspa.2009.0619.

Schultz-Tokos K.L., Hinrichsen H.H. and W. Zenk, 1994: Merging and migration of two meddies. - it J. Phys. Oceanogr., 24, 2129-2141.

Sokolovskiy M.A. and X. Carton, 2010: Baroclinic multipole formation from heton interaction. Fluid Dyn. Res., 42, 045501 (31 pp).

Sokolovskiy M.A. and J. Verron, 2000: Finite-core hetons: Stability and interactions. J. Fluid Mech., 423, 127-154.

Sokolovskiy M.A. and J. Verron, 2014: Dynamics of vortex structures in a stratified ro- 
tating fluid. Series Atmospheric and Oceanographic Sciences Library. Vol. 47, Springer: Switzerland, $382 \mathrm{pp}$.

Sutyrin G.G. and R. Grimshaw, 2010: The long-time interaction of an eddy with shelf topography. Ocean Model., 32, 25-35.

Valcke S. and J. Verron, 1997: Interactions of baroclinic isolated vortices: the dominant effect of shielding. J. Phys. Oceanogr., 27, 524-541.

Vandermeirsch F.O., Carton X,J, and Y.G. Morel, 2003: Interaction between an eddy and a zonal jet. Part I. One and a half layer model Dyn. Atmos. Oceans, 36, 247-270. Verron J. and S. Valcke, 1994: Scale-dependent merging of baroclinic vortices. J. Fluid Mech., 264, 81-106.

Vic C., Roullet G., Carton X. and X. Capet, 2014: Mesoscale dynamics in the Arabian Sea and a focus on the Great Whirl lifecycle: a numerical investigation using ROMS. J. Geophys. Res., 119, 9, 6422-6443.

Vic C., Roullet G. Capet X. and X. Carton, 2015: Eddy-topography interactions and the fate of the Persian Gulf outflow. J. Geophys. Res., 120, doi:10.1002/2015JC011033.

Yasuda I., 1995: Geostrophic vortex merger and streamer development in the ocean with special reference to the merger of Kuroshio warm-core rings. J. Phys. Oceanogr., 25, 979-996.

Yasuda, I. and G.R. Flierl, 1995: Two-dimensional asymmetric vortex merger: Contour dynamics experiments. J. Oceanogr., 51, 145-170.

Yasuda, I. and G.R. Flierl, 1997: Two-dimensional asymmetric vortex merger: merger dynamics and critical merger distance. Dyn. Atmos. Oceans, 26, 159-181.

Zhang Y., Pedlosky J. and G.R. Flierl, 2011: Shelf Circulation and Cross-Shelf Transport out of a Bay Driven by Eddies from an Open-Ocean Current. Part I: Interaction between a Barotropic Vortex and a Steplike Topography. J. Phys. Oceanogr., 41, 889-910. 